\title{
Dynamic Link Adaptation For Filterband Multicarrier In Networks With Diverse Service Quality And Throughput Requirements
}

\section{Amna Javed Tiwana}

NUST: National University of Sciences and Technology

\section{Muhammad Zeeshan}

NUST: National University of Sciences and Technology

\section{Tabinda Ashraf}

NUST: National University of Sciences and Technology

\section{Muhammad Umar Farooq}

NUST: National University of Sciences and Technology

\section{Kashif Shahzad}

NUST: National University of Sciences and Technology

Adnan Akhunzada ( $\square$ akhunzadaadnan@gmail.com )

Technical University of Denmark: Danmarks Tekniske Universitet https://orcid.org/0000-0001-83709290

\section{Research Article}

Keywords: Filterbank Multicarrier, Quality of Service, Link Adaptation

Posted Date: May 26th, 2021

DOl: https://doi.org/10.21203/rs.3.rs-371334/v1

License: (c) (i) This work is licensed under a Creative Commons Attribution 4.0 International License. Read Full License

Version of Record: A version of this preprint was published at Telecommunication Systems on November 6th, 2021. See the published version at https://doi.org/10.1007/s11235-021-00845-3. 


\title{
Dynamic Link Adaptation for Filterband Multicarrier in Networks with Diverse Service Quality and Throughput Requirements
}

\author{
Amna Javed Tiwana - Muhammad Zeeshan - Tabinda Ashraf • \\ Muhammad Umar Farooq · Kashif Shahzad · Adnan Akhunzada
}

Received: date / Accepted: date

\begin{abstract}
The fast evolution in wireless communication standards and enhancement in cellular applications has created an exponential rise in the data rate requirement over the past few decades. The next generation wireless standards, therefore, need not only to provide ultra high data rates with minimum latency, but also to support diverse quality-of-service (QoS) requirements. Filterbank multicarrier (FBMC) scheme provides quite a few advantages over the conventional orthogonal frequency division multiplexing (OFDM) for future wireless networks. In order to achieve adaptive throughput and diverse service quality requirements under varying channel conditions, a link adaptation algorithm for FBMC scheme is proposed in this paper. The aim is to provide the dynamic selection of optimum parameters resulting in diverse modes of operation to ensure the desired and/or best available communication service quality. The proposed link adaptation algorithm incorporates the parameters of required data rate, available channel condition and QoS demand, and dynamically allocates the appropriate FBMC transmission parameters. The proposed scheme has been evaluated for AWGN as well as multipath fading SUI channel models. Simulation results indicate that the proposed algorithm successfully achieves the desired service quality subject to the availability of suitable channel conditions and
\end{abstract}

A. J. Tiwana, M. Zeeshan, T. Ashraf, M. U. Farooq, K. Shahzad

College of Electrical \& Mechanical Engineering, National University of Sciences \& Technology, Islamabad, 44000, Pakistan E-mail: ranazeeshan@ceme.nust.edu.pk

A. Akhunzada

DTU Compute, Technical University of Denmark, Kongens.

Lyngby, Denmark

E-mail:dnak@dtu.dk is also superior to the existing algorithms in terms of block error rate and effective throughput.

Keywords Filterbank Multicarrier - Quality of Service · Link Adaptation

\section{Introduction}

The enhancement in the cellular applications and the evolution in wireless communication standards over the past few decades stipulate ultra-high data rates with minimum latency for next generation wireless communication networks $[1,2]$. The advent of the era of Software Defined Radio (SDR) has rendered possible the achievement of such high data rates [3-5]. Up until the last decade Orthogonal Frequency Division Multiplexing (OFDM) was considered the most promising wideband networking scheme for SDR waveform design providing with better spectral efficiency than simplistic Frequency Division Multiplexing (FDM) [6,7]. However, due to spectral leakage issues or large undesired Outof-Band emission, CP-OFDM had to be transformed into an evolved of FBMC/OQAM, allowing significant enhancement in spectral efficiency as well as latency of the system. The elimination of $\mathrm{CP}$, the transmitter and receiver filter banks and the utilization of Offset-QAM modulation results in ISI and ICI reduction ad in term provides improvement in system efficiency. [8-12].

With the constantly growing number of wireless communication users an efficient resource allocation of Radio Frequency (RF) spectrum has become a crucial factor to be considered for SDR platform. For a rather streamlined utilization of RF bandwidth the adaptation of transmission side parameters according to the available channel conditions i.e. Signal to Noise Ratio 
(SNR), the application at hand i.e. the required Quality of Service (QoS) and the required data rate needs to be observed. This process is termed as link adaptation and is considered pivotal strategy for SDR waveform design [13,14]. Adaptive Modulation and Coding (AMC) is a well-known technique to undergo link adaptation and a vast research has been carried out in this domain where modulation order and code rate are varied w.r.t. the fluctuating channel conditions [1517]. Currently, various AMC algorithms are available for either single carrier based i.e. Time Division Multiple Access (TDMA) or Code Division Multiple Access (TDMA) and/or multicarrier OFDM based [18-25]. An FBMC/OQAM based link adaptation algorithm utilizing the Low Density Parity Check (LDPC) codes has been presented in [26], where the performance analysis has done under AWGN environment without the consideration of user throughput requirements.

A novel OFDM based AMC algorithm that jointly handles the optimized selection of the AMC modes and the on-chip power management of buffering memories is presented in [27]. Thus in contrast to conventional AMC which realizes the wireless channel conditions only this research considers both the wireless channel conditions and the buffering memory status, and conserves upto $58 \%$ energy efficiency for memory sub-systems contrary to the orthodox perfect memory based algorithms. Although the proposed AMC algorithm enhances energyefficiency at the receiving end, it results in throughput degradation at the transmitting end. A Fuzzy Rule Based Inference (FRBS) link adaptation algorithm with hybrid adaptive Time Division Multiple Access (TDMA)/ Multicode Code Division Multiple Access (MCDMA) based wideband networking waveform of SDR was proposed in [21]. A constrained optimization problem is formulated and then solved by forming Fuzzy Rule Matrix $(\mathrm{FRM})$ in order to reduce packet re-transmission overhead in this paper. Another Fuzzy Inference Based AMC algorithm with the energy efficient underlying scheme of OFDM was proposed in [28], in which modulation order is optimized according to the values of SNR, BER, exiting modulation order and coding rate. The required throughput has not been considered in this paper. For FBMC/OQAM with low density parity check (LDPC) codes, a link adaptation scheme is proposed in [29]. This scheme is a modified version of the existing AMC scheme to accommodate the characteristics of non-binary LDPC codes. It doesn't takes the throughput requirement of users to perform link adaptation.

An improved energy efficiency and spectrum utilization in wireless communication systems was proposed in [23] where instead of utilizing rectangular QAM con- stellation with two-dimensional square portions, hexagonal QAM modulation in combination with non-binary coding was incorporated. Adaptive modulation in another research [24] is considered in order to calculate the cell coverage radius into a cell for different modulations schemes and also to guarantee a target mean BER. A Continuous Phase Modulation (CPM) based, novel algorithm for simultaneous transmission and reception of signals of multiple users a novel hybrid narrow-band/ wideband networking waveform for a heterogeneous SDR network is proposed most recently in [25].

Although the link adaptation techniques at hand provide with a vast variety of algorithms based on various energy proficient and spectrally efficient transmission schemes, but they do not incorporate the consideration of user throughput requirement along with a Filterbank Multicarrier based modulation for adaptive modulation and coding. In this paper, a human intuition based conditional algorithm for physical layer cognition with FBMC/OQAM based wideband networking waveform is proposed. To amend the effects of channel fluctuations and to consider the user requirements of quality of service and throughput, a cognition engine is devised, providing with a specific set of transmission parameters.

Following are the major contributions of this paper.

1. An in-depth parametric analysis of FBMC/OQAM scheme is done in both additive white gaussian noise (AWGN) and stanford university interim (SUI) channel models

2. Concept of cognition engine is developed to provide optimum transmission parameters and mode of operation.

3. A novel link adaptation scheme is proposed by taking the desired throughput into consideration in addition to the channel condition and service quality. A significant advantage is the reduction in complexity by limiting the throughput to the desired even if the channel is capable of providing higher data rates.

\section{System Overview and Problem Formulation}

The present-day progress in the remote correspondence as well as continuously increasing cellular application and users have overburdened the radio frequency spectrum. Alongside that, a portion of frequency ranges are considerably used and some are underutilized. To cater for these wasteful aspects of spectrum and inefficiencies, some of the current ideal models like Software Defined Radio and Cognitive Radio have been presented in the course of recent decades. Accordingly an extreme pre- 


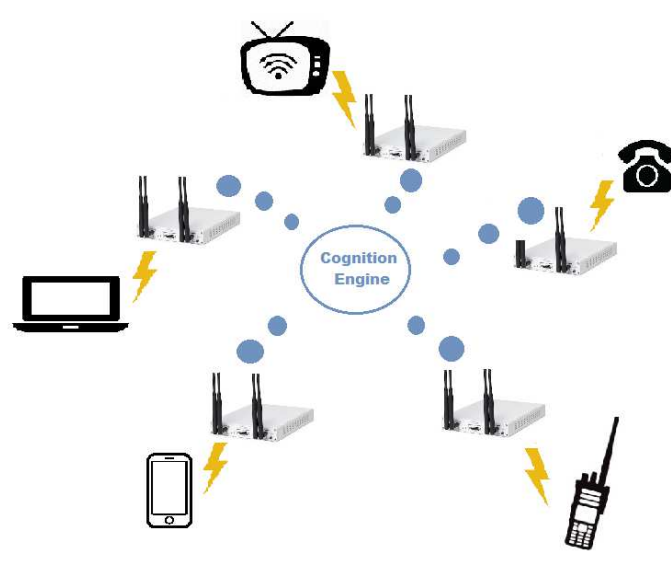

Fig. 1: Networked demonstration for various QoS applications

requisite of information rate that guarantees an ideal Quality of Service (QoS) for the given channel conditions has become a paramount need Fig. 1.

Considering that the present link adaptation algorithms are either MC-CDMA, adaptive TDMA or OFDM/F based with no consideration of user throughput requirement, the aim of this research is to develop an adaptive methodology for FBMC-based wideband waveform under fading channel environment. The research is directed towards the development of a link adaptation algorithm that provides with best possible throughput via a specific set of FBMC parameters for the required data rate, available Signal to Noise Ration (SNR) and maximum allowable Bit Error Rate (BER) or QoS.

\subsection{FBMC/OQAM System Model}

The FBMC framework is established on the Physical Layer for Dynamic Access and Cognitive Radio (PHYDAS) project [30] introduced in 2010 providing with the technical and theoretical aspects of prototype filter based Filterbank Multicarrier scheme in a well-organized and objective manner. The causal and real-valued, Finite Impulse Response (FIR) based prototype filter is the key constituent for Synthesis Filter Bank (SFB) and Analysis Filter Bank (AFB) which are the frequency shifted versions of low pass prototype frequency response [30-33]. A highly selective filter is obtained from the prototype filter response constituting $2 K-1$ pulses in the frequency domain with the help specifically defined frequency coefficients in [30]. Here, $K$ is the ratio of filter impulse impulse response to the multicarrier symbol duration or the number of samples the overlap in the time domain and is termed as the Overlapping factor utilized for characterizing the prototype filter. The impulse response of this prototype filter is provided as follows in the equation 1.

$h(m)=1+2 \sum_{k=1}^{K-1}(-1)^{k} H(k) \cos \left(\frac{2 \pi k m}{L}\right)$

here,

$m=1,2,3, \ldots, L-1$

$L$ is the prototype filter length and could be of any choice among $L=K M, L=K M+1$ or $L=K M-1$, where the total number of subcarriers are given by $M$. The utilized FBMC transmitter-receiver block diagram with channel encoding, OQAM modulation, filter banks and equalization is shown in the Fig. 2.

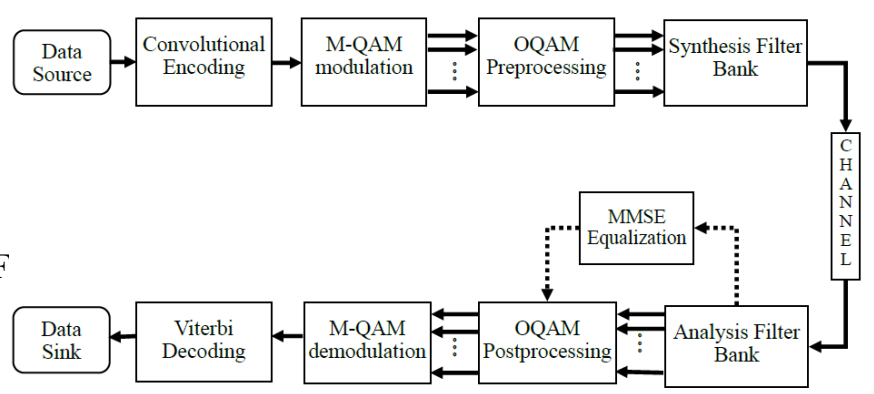

Fig. 2: FBMC/OQAM - System Model

After the addition of redundancy in the source data with the help of convolutional encoding, the data is modulated with QAM modulation. Subsequently the serial to parallel conversion and OQAM modulation by segregating complex modulated symbols into real and imaginary components in order to gain overall exploitation of channel bandwidth $[34,35]$ is done. The complex input symbols are given as,

$s_{n}[i]=s_{n}^{I}[i]+s_{n}^{Q}[i]$

where, $s_{n}[i]$ is the complex QAM symbol, $s_{n}^{I}[i]$ is the real or in-phase part and $s_{n}^{Q}[i]$ is the imaginary or quadrature part. These components can be further elaborated as,

$$
\begin{aligned}
& s_{n}^{I}[i]=\sum_{n=0} s_{n}^{I}[i] \delta(t-n T) \\
& s_{n}^{Q}[i]=\sum_{n=0} s_{n}^{Q}[i] \delta(t-n T)
\end{aligned}
$$

Finally the modulated data symbols are passed through IFFT based SFB and sent over the multipath fading 
channel. The fading environment has been realized by utilizing real-time rician distribution based Stanford University Interim (SUI) fading channels [36]. At the receiving end, the noisy data from the frequency selective fading channel is first passed through the FFT based AFB and then equalized with MMSE equalizer in order to cater for the effects of the channel. Following the equalization OQAM post processing is done where the data that was split into real and complex parts is recombined again and converted back to serial data from the incoming parallel streams. In the end the data is decoded utilizing the Viterbi decoder as a counterpart of convolutional encoding employed on the transmitting side.

\subsection{Problem Formulation}

Firstly the throughput problem under consideration is presented based upon the channel conditions as well as the user requirements. The throughput is calculated with the involving the parameters of modulation order, subcarrier count, symbol time, encoding rate and the available RF bandwidth.

$R_{k}=\frac{\log _{2}\left(M_{k}\right) N r}{T_{\text {sym }}}$

where,

$R_{k}=$ Throughput for $k^{t h}$ user in Mbps

$M_{k}=$ Modulation order for $k^{t h}$ user

$\log _{2}\left(M_{k}\right)=$ Bits per OQAM symbol for the $k^{t h}$ user

$N_{k}=$ Number of subcarriers

$r_{k}=$ Code rate

$T_{\text {sym }}=$ Symbol duration

Incorporating the subcarrier count and RF bandwidth, symbol duration is given as follows,

$T_{\text {sym }}=\frac{N}{B_{R F}}$

The overall problem is thus to maximize the user throughput as,

$\max _{M_{k}, N_{k}, r_{k}} R_{k}=\log _{2}\left(M_{k}\right) r B_{R F}$

subjected to the constraints,

$$
\mathrm{BER} \leq \mathrm{BER}_{\max }
$$

$R_{k}=\left\{\begin{array}{cll}R_{\max } & \text { if } & R_{\max } \leq R_{r e q} \\ R_{r e q} & \text { if } \quad R_{\max }>R_{r e q}\end{array}\right.$

here,

$\mathrm{BER}=$ Bit Error Rate

$\mathrm{BER}_{\max }=$ Maximum allowed BER as per QoS demand

$R_{k, r e q}=$ Throughput demand of $k^{t h}$ user (Mbps)

$R_{\max }=$ Maximum possible throughput

The aim is to provide maximum throughput to the user, if the required throughput is more than or equal to the maximum available throughput. On the other hand, if the required throughput is less than the maximum available throughput, the data rate is restricted to the required user throughput in order to conserve computational complexity and power consumption even if channel conditions are well enough to support better throughput. The calculated symbol duration and throughput are listed in the tables 2 and 3 respectively. The BER range is considered to be between $10^{-4} \leq$ $\mathrm{BER}_{\max } \leq 10^{-1}$ and the data rates vary within a range of $2.5 \mathrm{Mbps} \leq R_{k, r e q} \leq 10 \mathrm{Mbps}$, with the RF bandwidth assumed to be at $5 \mathrm{MHz}$. The utilized notations as well as their respective ranges are given in the table 1.

\section{Parametric Performance Analysis}

In order to establish the link adaption scheme properly, the first phase is the performance and parametric analysis of the FBMC/OQAM system under various parameters. An in-depth analysis is performed for all 6 SUI fading channel models as well as AWGN.

Performance analysis is carried out by examining the effects of convolutional encoding and Offset-QAM modulation within the system model, under four different scenarios: FBMC/QAM without encoding, FBMC/QAM with encoding, FBMC/OQAM without encoding and FBMC/OQAM with encoding.

\subsection{FBMC/QAM without encoding}

The first case is the basic FBMC framework with single input single output (SISO) model based upon Quadrature Amplitude modulation for 256 subcarriers and an overlapping factor $K=4$. The $\frac{E_{b}}{N_{0}}$ is set for the range of $[-5,25] d B$, resulting in SNR range as, 
Table 1: Input and output variable values and ranges

\begin{tabular}{cc}
\hline Parameters & Values/Ranges \\
\hline QoS Values: $E_{1}, E_{2}, E_{3}, E_{4}$ & $10^{-1}, 10^{-2}, 10^{-3}, 10^{-4}$ \\
\hline SNRs: $S_{1}, S_{2}, S_{3}, S_{4}$ & For AWGN: $[-5,-2],[-2,1],[1,4],[4,7] \mathrm{dB}$ \\
\cline { 2 - 2 } & For SUI-4: $[-3,4],[4,11],[11,18],[18,25] \mathrm{dB}$ \\
\hline Throughput: $R_{1}, R_{2}, R_{3}, R_{4}$ & {$[2,4],[4,6],[6,8],[8,10] \mathrm{Mbps}$} \\
\hline
\end{tabular}

Table 2: Calculated Symbol Duration

\begin{tabular}{cc}
\hline Subcarrier Count & Symbol Time (sec) \\
\hline 128 & $2.56 e^{-5}$ \\
\hline 256 & $5.12 e^{-5}$ \\
\hline 512 & $1.024 e^{-4}$ \\
\hline
\end{tabular}

Table 3: Calculated Data Rates for Link Adaptation

\begin{tabular}{|c|c|c|}
\hline Modulation Order & Encoding rate & $R_{k}(\mathrm{Mbps})$ \\
\hline \multirow{3}{*}{2} & 1 & 5 \\
\hline & $1 / 2$ & 2.5 \\
\hline & $2 / 3$ & 3.33 \\
\hline \multirow{3}{*}{4} & 1 & 10 \\
\hline & $1 / 2$ & 5 \\
\hline & $2 / 3$ & 6.67 \\
\hline
\end{tabular}

$S N R(d B)=\frac{E_{b}}{N_{0}}+10 \log _{10}(b r)$

where, $b$ is bits per subcarrier and $r$ is the convolutional encoding rate. For performance analysis, the modulation order is set as 4 and no channel encoding has been incorporated or in other words the rate is set to 1 . The BER curves for AWGN and SUI channels are shown in the Fig. 3.

\subsection{FBMC/QAM with encoding}

In order to provide immunity towards multipath channel noise, convolutional encoder is utilized in order to add redundancy to the original data according to some specific rule [37-39]. Convolutional encoder allows the discrete type convolution of the impulse responses of both the input and the encoder. The error correction code rule that we have utilized for this case is $1 / 2$, i.e. for every single bit fed into the encoder, 2 output bits are generated. BER curve for this case is shown in the

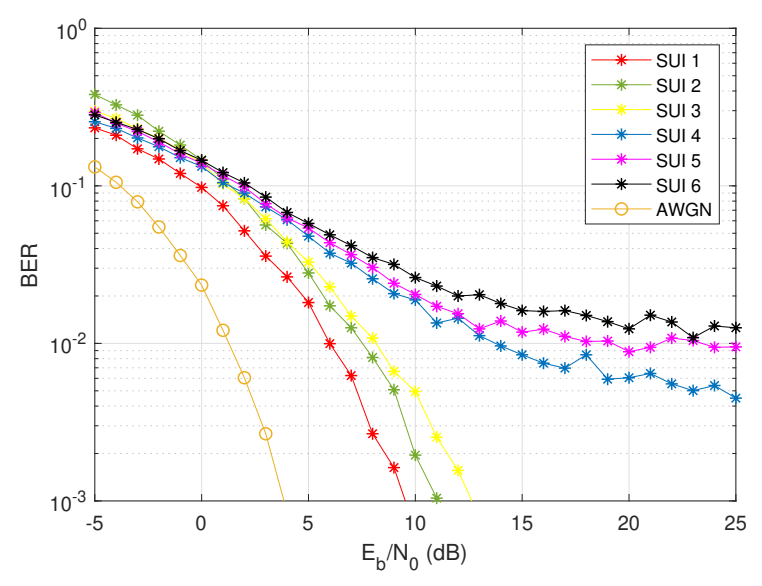

Fig. 3: FBMC/QAM without convolutional encoding

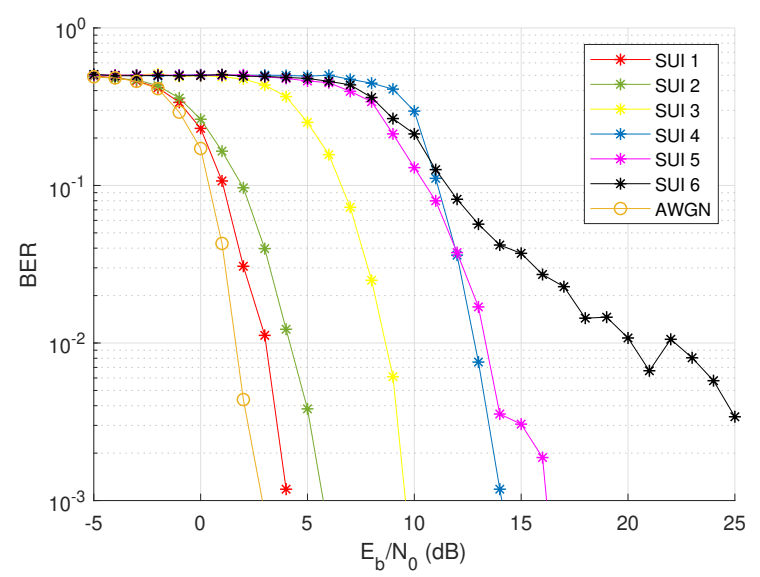

Fig. 4: FBMC/QAM with convolutional encoding

Fig. 4 keeping the constraint length i.e. delay for the input bit streams to the encoder, at 7 and soft decoding is done via Viterbi decoder [40]. Punctured convolutional coding is utilized for the code rate $2 / 3$ in order to reduce computational complexity and is derived from the mother code of rate $1 / 2[41,42]$ 


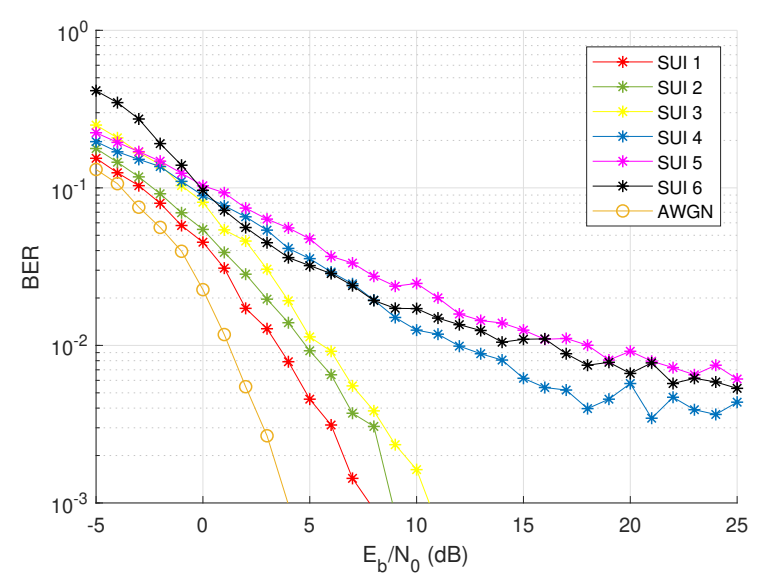

Fig. 5: FBMC/OQAM without convolutional encoding

\subsection{FBMC/OQAM without encoding}

The utilization of Offset-QAM instead of QAM ensures further that the FBMC symbol overlaps with the neighboring symbol only bringing the neighbor orthogonality to the system. Staggering QAM symbols by half the symbol period $(T / 2)$ ensures that the real and imaginary parts of the data are not transmitted at the same time as in OFDM, helping avoid a huge phase shift, and thus avoiding the zero-crossing to occur whenever both the bits change simultaneously. In this way huge spectral side lobes can be avoided and the condition of orthogonality is further strengthened [34,35,43]. The BER curves for this case are shown in the Fig. 5.

\subsection{FBMC/OQAM with encoding}

Finally both the modifications i.e. convolutional encoding along with Offset-QAM modulation, are combined in case 4 , in order to obtain maximum orthogonality and spectral efficiency. It should be noted that the basic SISO model remains same for all the simulations. The curves in final scenario are depicted in the Fig. 6.

The overall performance comparison is summarized in the Table 4, depicting the minimum SNR value required to achieve a QoS corresponding to BER of $10^{-2}$ for all scenarios under 6 SUI channels. The results clearly indicate the improved BER curves for the last case and worst for first. The performance deteriorates significantly when going from SUI-1 upto SUI-6 as the channel is transcending from light tree density having lesser multipath fading effects towards heavy tree density involving larger interference.

In order to select appropriate parameters for transmission, parametric analysis of FBMC/OQAM is carried out in SUI-4 channel model by varying modulation

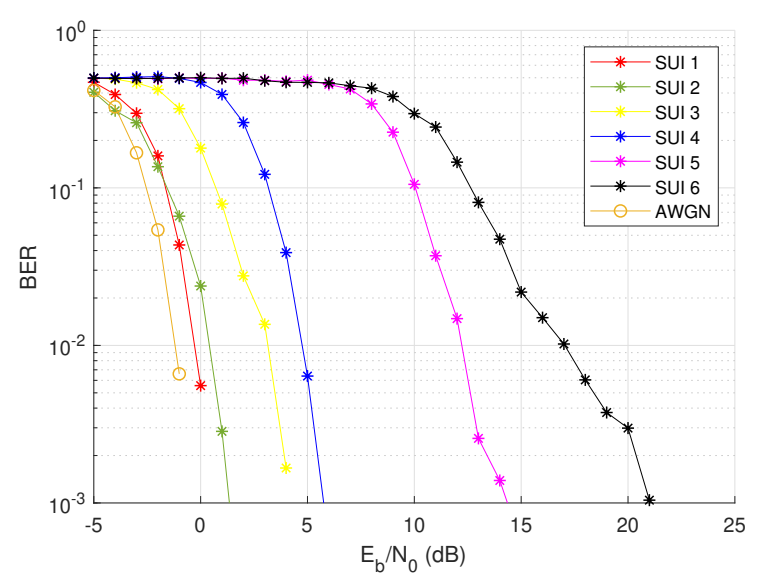

Fig. 6: FBMC/OQAM with convolutional encoding

order, overlapping factor, number of subcarriers and encoding rate. The utilized parameters are summarized in the Table 5. Threshold value of BER is taken as $10^{-2}$ and the minimum SNR values to attain this QoS are shown in table 6 . The increment in the overlapping factor and number of subcarriers improve BER performance. Having a large value of overlapping factor depicts longer filter impulse response duration extending it over to various symbols and thus, improving the BER. Although the decoder efficiency increases with the constrained length, however, large constraint length results in efficient encoding [38]. The constraint length is kept to 7 for both code rates of $1 / 2$ and $2 / 3$.

\section{Proposed Link Adaptation}

In this section, we present the proposed link adaption algorithm consisting of a cognitive engine (CE). The proposed cognitive engine (see figure 7 ) is a facilitating block of intelligence between physical layer and MAC. It ensures the maximal utilization of physical layer to satisfy several service parameters. Based on the underlying channel conditions and user requirements of QoS and throughput, it chooses suitable transmission parameters constituting a mode of operation. The complete process is comprised of the following two stages:

1. Data acquisition from BER curves

2. Adaptive selection of transmission parameters

\subsection{Data Acquisition from BER curves}

The appropriate selection of parameters to fulfill QoS and throughput demands require acquisition of data from BER curves. For this, we need to obtain BER 
Table 4: Performance Analysis of FBMC under SUI Channel Models with different scenarios

\begin{tabular}{cccccccc}
\hline Case No. & AWGN & SUI-1 & SUI-2 & SUI-3 & SUI-4 & SUI-5 & SUI-6 \\
\hline 1 & 1.319 & 5.793 & 7.481 & 8.52 & 13.72 & 18.07 & 23.07 \\
\hline 2 & 1.642 & 3.041 & 4.165 & 8.152 & 12.8 & 13.34 & 20.15 \\
\hline 3 & 1.191 & 3.506 & 5.008 & 5.863 & 12.04 & 16.02 & 15.03 \\
\hline 4 & -1.207 & -0.2768 & 0.4017 & 3.16 & 4.752 & 12.22 & 17.06 \\
\hline
\end{tabular}

Table 5: Simulation Parameters for Parametric Analysis

\begin{tabular}{cc}
\hline Parameters & Values \\
\hline Modulation Order & 2OQAM, 4OQAM \\
\hline Number of Subcarrier & $128,256,512$ \\
\hline Overlapping Factor & $2,3,4$ \\
\hline Encoding Rate & $1 / 2,2 / 3$ \\
\hline
\end{tabular}

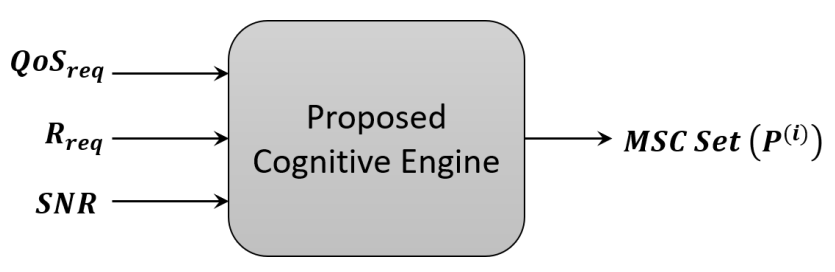

Fig. 7: Concept of cognitive engine

curves for FBMC/OQAM by using all possible and chosen values of modulation order, subcarrier count and code rate. We have selected modulation order of 2 and 4 , subcarrier count of 128,256 and 512, and code rate of $1,1 / 2$ and $2 / 3$. This results in a total of 18 modes of operation. Each parameter set is denoted by MSC set (i.e. modulation order, subcarrier count, code rate). All these 18 modes of operation in the form of MSC set are represented in the Table 7 .

The data is then acquired from BER curves obtained under AWGN and SUI-4 channel models for all the possible modes of operation listed in the Table 7. The data is acquired from the intersection point obtained by drawing a horizontal line under each QoS for all the performance curves. As an instance, the data acquisition for three modes $P_{1}, P_{6}$ and $P_{13}$ under AWGN and SUI-4 channels are shown in the Fig. 8 and Fig. 9 , respectively.

The performance for $P_{1}$ is the worst as the convolutional encoding for this mode is 1 i.e. uncoded where as best case here is obtained when number of subcarriers are increased to 512 i.e. mode $P_{6}$. As there are no multipath fading effects in the case of AWGN, the increase in subcarrier count has a minute effect on performance. The variations are more apparent in SUI-4 case.

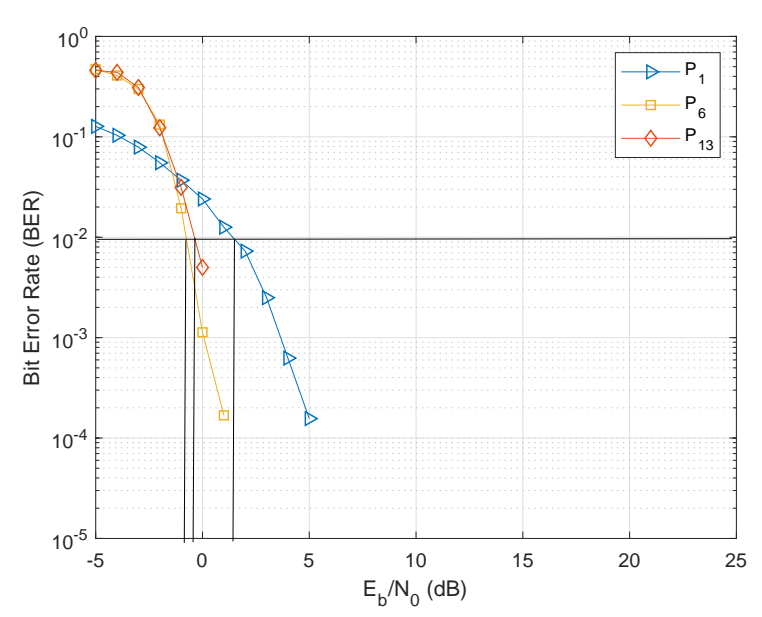

Fig. 8: Data Acquisition for various modes of operation in AWGN

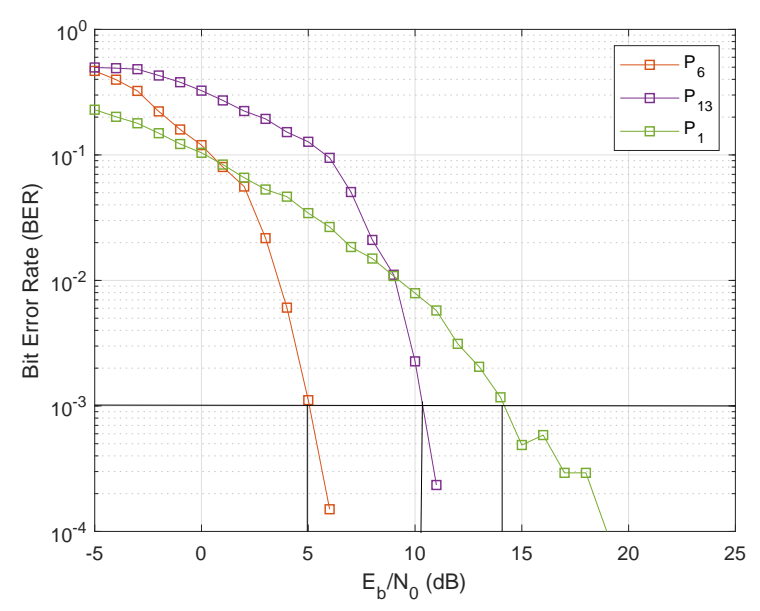

Fig. 9: Data Acquisition for various modes of operation in SUI-4

The acquired data indicates the minimum value of SNR guaranteeing the BER to be within maximum allowable value along with the achievable throughput for a specific mode of operation (MSC set). The acquired data for AWGN and SUI-4 cases are given in three dimensional tables as there are three parameters under consideration. For AWGN case, the SNR values for 
Table 6: Parametric Analysis of FBMC under SUI Channel Models with various Parameters

\begin{tabular}{ccccccccc}
\hline Parameters & & AWGN & SUI-1 & SUI-2 & SUI-3 & SUI-4 & SUI-5 & SUI-6 \\
\hline \multirow{2}{*}{ Modulation Order } & 2 & -1.401 & -0.738 & 1.448 & 2.102 & 6.528 & 10.46 & 13.63 \\
\cline { 2 - 8 } & 4 & -1.217 & -0.2768 & 2.327 & 4.305 & 7.201 & 12.22 & 16.17 \\
\hline \multirow{3}{*}{ Number of Subcarriers } & 128 & -1.028 & 1.868 & 4.463 & 6.381 & 8.143 & 10.42 & 19.62 \\
\cline { 2 - 8 } & 256 & -1.217 & -0.2768 & 2.327 & 4.305 & 7.201 & 9.962 & 16.17 \\
\cline { 2 - 8 } & 512 & -1.341 & -0.8858 & 1.766 & 2.601 & 5.697 & 8.922 & 10.06 \\
\hline \multirow{2}{*}{ Overlapping Factor } & 2 & -1.045 & 1.955 & 6.281 & 7.559 & 11.32 & 15.48 & 22.84 \\
\cline { 2 - 8 } & 3 & -1.089 & 1.137 & 3.259 & 6.238 & 10.23 & 13.81 & 18.36 \\
\hline Encoding Rate & $1 / 2$ & -1.217 & -0.2768 & 2.327 & 4.305 & 7.201 & 12.22 & 16.17 \\
\cline { 2 - 8 } & $2 / 3$ & -0.4791 & -0.6101 & 3.823 & 7.196 & 8.512 & 14.95 & 19.48 \\
\hline
\end{tabular}

Table 7: Output MSC (Modulation order, Subcarrier count, Code rate) pairs for 18 set of values

\begin{tabular}{cccc}
\hline Value & MSC Pair & Value & MSC Pair \\
\hline$P_{1}$ & $\{2,128,1\}$ & $P_{10}$ & $\{4,128,1\}$ \\
\hline$P_{2}$ & $\{2,256,1\}$ & $P_{11}$ & $\{4,256,1\}$ \\
\hline$P_{3}$ & $\{2,512,1\}$ & $P_{12}$ & $\{4,512,1\}$ \\
\hline$P_{4}$ & $\{2,128,2 / 3\}$ & $P_{13}$ & $\{4,128,2 / 3\}$ \\
\hline$P_{5}$ & $\{2,256,2 / 3\}$ & $P_{14}$ & $\{4,256,2 / 3\}$ \\
\hline$P_{6}$ & $\{2,512,2 / 3\}$ & $P_{15}$ & $\{4,512,2 / 3\}$ \\
\hline$P_{7}$ & $\{2,128,1 / 2\}$ & $P_{16}$ & $\{4,128,1 / 2\}$ \\
\hline$P_{8}$ & $\{2,256,1 / 2\}$ & $P_{17}$ & $\{4,256,1 / 2\}$ \\
\hline$P_{9}$ & $\{2,512,1 / 2\}$ & $P_{18}$ & $\{4,512,1 / 2\}$ \\
\hline
\end{tabular}

Table 8: Data Acquisition for $M=2$ in AWGN

\begin{tabular}{|c|c|c|c|c|}
\hline $\mathbf{N}$ & BER & $r=1$ & $r=2 / 3$ & $r=1 / 2$ \\
\hline \multirow{4}{*}{128} & $E_{1}$ & -3.876 & -1.716 & -2.743 \\
\hline & $E_{2}$ & 1.183 & -0.3463 & -1.268 \\
\hline & $E_{3}$ & 3.607 & - & -0.09797 \\
\hline & $E_{4}$ & - & - & - \\
\hline \multirow{4}{*}{256} & $E_{1}$ & -3.897 & -1.807 & -2.745 \\
\hline & $E_{2}$ & 1.052 & -0.5708 & -1.245 \\
\hline & $E_{3}$ & 3.634 & 0.5355 & -0.3204 \\
\hline & $E_{4}$ & 4.648 & - & - \\
\hline \multirow{4}{*}{512} & $E_{1}$ & -3.924 & -1.937 & -2.597 \\
\hline & $E_{2}$ & 1.332 & -0.5848 & -1.325 \\
\hline & $E_{3}$ & 3.794 & - & -0.3665 \\
\hline & $E_{4}$ & 5.351 & - & - \\
\hline
\end{tabular}

$M=2$ and $M=4$ are given in the tables 8 and 9 . For SUI-4 case, the SNR values for $M=2$ and $M=4$ are given in the tables 10 and 11 .

\subsection{Adaptive Selection of Transmission Parameters}

In this section, we present the proposed algorithm for adaptive selection of transmission parameters. The proposed algorithm takes required throughput, QoS and the channel SNR as input and provides with the most appropriate transmission parameters or MSC set. It comprises of a collection of logic rules in the form of IF-THEN statements. A total of 64 rules are devised based on either concurring rules or conflicting rules. The selected modes of operation for AWGN and SUI-4 are shown in the Table 12 and Table 13, respectively.

The rules that proceed without any conflict between antecedent i.e. IF and consequent i.e. THEN are termed as concurring rules while the rules with a contradiction between the two are conflicting rules. The algorithm starts by taking two inputs i.e. the available SNR and required QoS by the user. If there exist some modes of 
Table 9: Data Acquisition for $M=4$ in AWGN

\begin{tabular}{|c|c|c|c|c|}
\hline $\mathbf{N}$ & BER & $r=1$ & $r=2 / 3$ & $r=1 / 2$ \\
\hline \multirow{4}{*}{128} & $E_{1}$ & -3.816 & -1.858 & -2.576 \\
\hline & $E_{2}$ & 1.309 & -0.5682 & -1.12 \\
\hline & $E_{3}$ & 3.721 & - & -0.1174 \\
\hline & $E_{4}$ & 5.412 & - & - \\
\hline \multirow{4}{*}{256} & $E_{1}$ & -3.992 & -1.883 & -2.664 \\
\hline & $E_{2}$ & 1.336 & -0.6017 & -1.134 \\
\hline & $E_{3}$ & 3.659 & 0.2375 & -0.1663 \\
\hline & $E_{4}$ & 5.354 & - & - \\
\hline \multirow{4}{*}{512} & $E_{1}$ & -3.957 & -1.852 & -2.588 \\
\hline & $E_{2}$ & 1.196 & -0.5561 & -1.216 \\
\hline & $E_{3}$ & 3.779 & 0.2271 & -0.354 \\
\hline & $E_{4}$ & 5.33 & 0.7478 & - \\
\hline
\end{tabular}

Table 10: Data Acquisition for $M=2$ in SUI-4

\begin{tabular}{|c|c|c|c|c|}
\hline $\mathbf{N}$ & BER & $r=1$ & $r=2 / 3$ & $r=1 / 2$ \\
\hline \multirow{4}{*}{128} & $E_{1} 0.1162$ & 2.242 & 3.217 & \\
\hline & $E_{2}$ & 9.307 & 6.654 & 6.366 \\
\hline & $E_{3}$ & 15.11 & - & - \\
\hline & $E_{4}$ & - & - & - \\
\hline \multirow{4}{*}{256} & $E_{1}$ & 0.7874 & 3.294 & 2.853 \\
\hline & $E_{2}$ & 6.099 & 5.103 & 4.833 \\
\hline & $E_{3}$ & 9.572 & 6.318 & 5.531 \\
\hline & $E_{4}$ & - & - & - \\
\hline \multirow{4}{*}{512} & $E_{1}$ & -2.602 & 0.4634 & -0.4786 \\
\hline & $E_{2}$ & 4.43 & 3.796 & 3.158 \\
\hline & $E_{3}$ & 8.723 & - & 4.384 \\
\hline & $E_{4}$ & 13.57 & - & 5.508 \\
\hline
\end{tabular}

Table 11: Data Acquisition for $M=4$ in SUI-4

\begin{tabular}{|c|c|c|c|c|}
\hline $\mathbf{N}$ & BER & $r=1$ & $r=2 / 3$ & $r=1 / 2$ \\
\hline \multirow{4}{*}{128} & $E_{1}$ & 0.8662 & 6.098 & 3.489 \\
\hline & $E_{2}$ & 11.81 & 9.329 & 7.982 \\
\hline & $E_{3}$ & 21.79 & 12.83 & 8.909 \\
\hline & $E_{4}$ & - & - & - \\
\hline \multirow{4}{*}{256} & $E_{1}$ & 0.1619 & 3.115 & 2.626 \\
\hline & $E_{2}$ & 7.704 & 6.122 & 5.229 \\
\hline & $E_{3}$ & 17.97 & 7.332 & - \\
\hline & $E_{4}$ & - & - & - \\
\hline \multirow{4}{*}{512} & $E_{1}$ & -2.305 & 0.4095 & -0.3931 \\
\hline & $E_{2}$ & 4.477 & 3.921 & 3.162 \\
\hline & $E_{3}$ & 9.037 & 5.09 & 4.525 \\
\hline & $E_{4}$ & 12.94 & - & - \\
\hline
\end{tabular}

operation that lie within this $E$ and $S$ bracket, then the third input of required data rate is given. After the final input if a single mode is extracted from this bracket, it would be considered as the optimum mode of operation and such rules would be termed as concurring rules.
On the other hand, the conflicting rules incorporate diverse consequent possibilities, such as if there are no modes within the bracket of $E$ and $S$ with the required QoS, the optimum mode would be considered the mode that is previously available providing with the best available QoS. Similarly, if there is no mode 


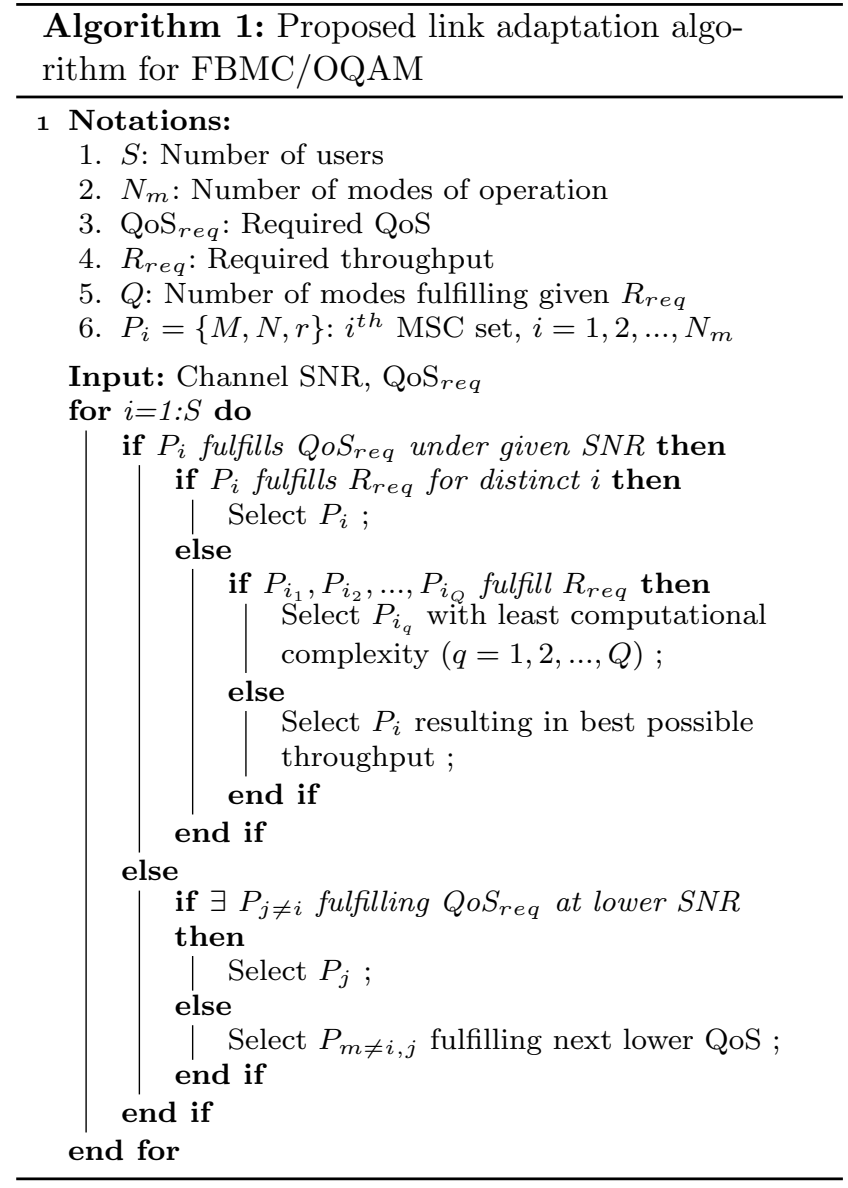

meeting the required SNR, the mode available at the lower SNR would be considered. This is because if a mode is running at a lower SNR, it would work for a higher vale of SNR as well. After the third input of data rate, if a single mode is not obtained from the $E, S$ and $R$ bracket, there could be two cases. If the bracket is providing no mode that is fulfilling the required data rate, the mode that is granting maximum throughput should be considered optimum. Contrarily, if there are more than one mode within the bracket after the third input, the computational complexity difference is observed among all the multiple modes. The overall complexity would rely upon the transmitting end parameters, such as, subcarrier count, order of modulation, convolutional code rate and Viterbi decoder configuration. In our case considering the parameters, increase in the modulation order or the number of subcarriers, the computational complexity would be enhanced [44]. The convolutional encoding, on the other hand, has a negligible effect in case of code rate $2 / 3$ due to enhanced trace-back length because of utilizing puncturing for Viterbi Decoder [45]. If a significant complexity difference is present, the mode with the least complexity should be considered best. While on account of negligi- ble computational complexity difference, the mode providing better throughput is extracted from the bracket.

Table 12: Conditional Rules for AWGN Channel

\begin{tabular}{cccccc}
\hline \multirow{2}{*}{ QoS } & SNR & $R_{1}$ & $R_{2}$ & $R_{3}$ & $R_{4}$ \\
\hline \multirow{4}{*}{$E_{1}$} & $S_{1}$ & $P_{7}$ & $P_{1}$ & $P_{10}$ & $P_{10}$ \\
\cline { 2 - 6 } & $S_{2}$ & $P_{4}$ & $P_{13}$ & $P_{13}$ & $P_{13}$ \\
\cline { 2 - 6 } & $S_{3}$ & $P_{4}$ & $P_{13}$ & $P_{13}$ & $P_{13}$ \\
\cline { 2 - 6 } & $S_{4}$ & $P_{4}$ & $P_{13}$ & $P_{13}$ & $P_{13}$ \\
\hline \multirow{4}{*}{$E_{2}$} & $S_{1}$ & $P_{7}$ & $P_{1}$ & $P_{10}$ & $P_{10}$ \\
\cline { 2 - 6 } & $S_{2}$ & $P_{4}$ & $P_{16}$ & $P_{13}$ & $P_{13}$ \\
\cline { 2 - 6 } & $S_{3}$ & $P_{10}$ & $P_{1}$ & $P_{10}$ & $P_{10}$ \\
\cline { 2 - 6 } & $S_{4}$ & $P_{10}$ & $P_{1}$ & $P_{10}$ & $P_{10}$ \\
\hline \multirow{5}{*}{$E_{3}$} & $S_{1}$ & $P_{7}$ & $P_{1}$ & $P_{10}$ & $P_{10}$ \\
\cline { 2 - 6 } & $S_{2}$ & $P_{5}$ & $P_{16}$ & $P_{14}$ & $P_{14}$ \\
\cline { 2 - 6 } & $S_{3}$ & $P_{10}$ & $P_{1}$ & $P_{10}$ & $P_{10}$ \\
\cline { 2 - 6 } & $S_{4}$ & $P_{10}$ & $P_{1}$ & $P_{10}$ & $P_{10}$ \\
\hline \multirow{3}{*}{$E_{4}$} & $S_{1}$ & $P_{7}$ & $P_{1}$ & $P_{10}$ & $P_{10}$ \\
\cline { 2 - 6 } & $S_{2}$ & $P_{15}$ & $P_{15}$ & $P_{15}$ & $P_{15}$ \\
\cline { 2 - 6 } & $S_{3}$ & $P_{15}$ & $P_{15}$ & $P_{15}$ & $P_{15}$ \\
\cline { 2 - 6 } & $S_{4}$ & $P_{15}$ & $P_{2}$ & $P_{10}$ & $P_{10}$ \\
\hline
\end{tabular}

Table 13: Conditional Rules for SUI-4 Channel

\begin{tabular}{cccccc}
\hline QoS & SNR & $R_{1}$ & $R_{2}$ & $R_{3}$ & $R_{4}$ \\
\hline \multirow{4}{*}{$E_{1}$} & $S_{1}$ & $P_{7}$ & $P_{1}$ & $P_{14}$ & $P_{10}$ \\
\cline { 2 - 6 } & $S_{2}$ & $P_{7}$ & $P_{1}$ & $P_{13}$ & $P_{10}$ \\
\cline { 2 - 6 } & $S_{3}$ & $P_{7}$ & $P_{1}$ & $P_{13}$ & $P_{10}$ \\
\cline { 2 - 6 } & $S_{4}$ & $P_{7}$ & $P_{1}$ & $P_{13}$ & $P_{10}$ \\
\hline \multirow{3}{*}{$E_{2}$} & $S_{1}$ & $P_{6}$ & $P_{18}$ & $P_{15}$ & $P_{15}$ \\
\cline { 2 - 6 } & $S_{2}$ & $P_{7}$ & $P_{1}$ & $P_{13}$ & $P_{11}$ \\
\cline { 2 - 6 } & $S_{3}$ & $P_{7}$ & $P_{1}$ & $P_{13}$ & $P_{10}$ \\
\cline { 2 - 6 } & $S_{4}$ & $P_{7}$ & $P_{1}$ & $P_{13}$ & $P_{10}$ \\
\hline \multirow{3}{*}{$E_{3}$} & $S_{1}$ & $P_{6}$ & $P_{18}$ & $P_{15}$ & $P_{15}$ \\
\cline { 2 - 6 } & $S_{2}$ & $P_{8}$ & $P_{2}$ & $P_{14}$ & $P_{12}$ \\
\cline { 2 - 6 } & $S_{3}$ & $P_{8}$ & $P_{1}$ & $P_{13}$ & $P_{11}$ \\
\cline { 2 - 6 }$E_{4}$ & $S_{4}$ & $P_{8}$ & $P_{1}$ & $P_{13}$ & $P_{10}$ \\
\cline { 2 - 6 } & $S_{1}$ & $P_{6}$ & $P_{18}$ & $P_{15}$ & $P_{15}$ \\
\cline { 2 - 6 } & $S_{2}$ & $P_{9}$ & $P_{18}$ & $P_{15}$ & $P_{15}$ \\
\cline { 2 - 6 } & $S_{3}$ & $P_{9}$ & $P_{3}$ & $P_{15}$ & $P_{12}$ \\
\cline { 2 - 6 } & $S_{4}$ & $P_{9}$ & $P_{3}$ & $P_{15}$ & $P_{12}$ \\
\hline & & & & &
\end{tabular}

\section{Simulation Results}

For the validation of the proposed link adaptation algorithm, we first preset the simulation results to demonstrate the effectiveness of the proposed algorithm to achieve the desired QoS and throughput requirements. Two scenarios under SUI-4 model with the given QoS 
and throughput requirements under specific channel conditions. For a QoS demand of $10^{-2}$, available SNR of $S_{2}$ i.e. $4 \mathrm{~dB}$ to $11 \mathrm{~dB}$ and a throughput requirement of 6.5 Mbps, the selected mode from the proposed algorithm is $P_{13}=\{4,128,2 / 3\}$. The minimum SNR required to achieve this QoS is $9.053 \mathrm{~dB}$, as indicated by the BER curve shown in the Fig. 10 for this mode of operation. Furthermore, throughput for $P_{13}$ is $6.67 \mathrm{Mbps}$, which is within the required user throughput.

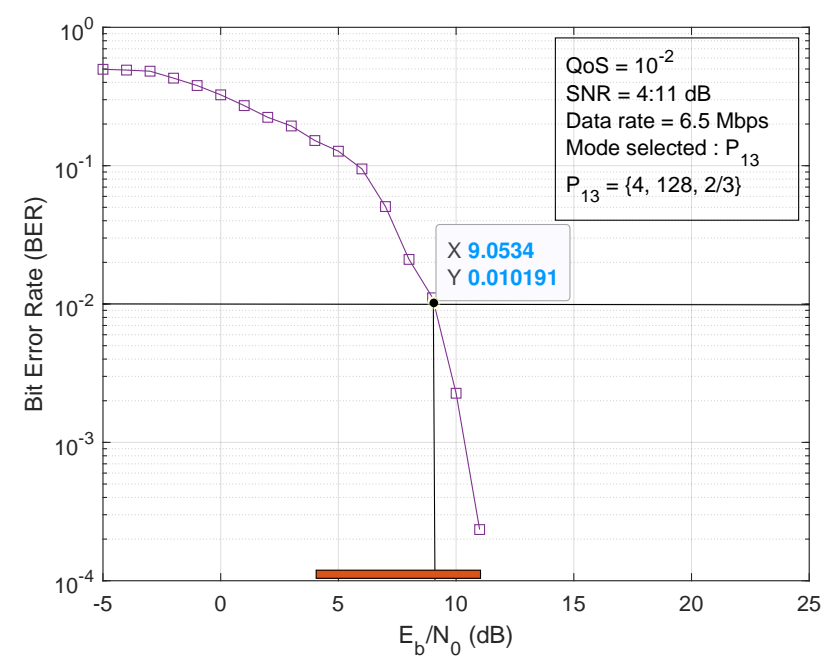

Fig. 10: Mode selection for $E_{2}, S_{2}, R_{3}$

Now we discuss a scenario where the available channel conditions are much better, indicated by SNR range of $18 \mathrm{~dB}$ to $25 \mathrm{~dB}$. The QoS and throughput demands are $10^{-3}$ and $2 \mathrm{Mbps}$, respectively. The proposed algorithm selects mode $P_{8}=\{2,256,1 / 2\}$, that ensures required QoS at an SNR of $5.898 \mathrm{~dB}$ (see figure 11). Even though the channel conditions here are fair enough to allow higher data rates, the proposed algorithm selects appropriate mode of operation by limiting the throughput to $2.5 \mathrm{Mbps}$. As mentioned earlier, this is done to reduce the computational complexity and power consumption. Hence, the proposed algorithm successfully allocates suitable mode of operation to satisfy the QoS and throughput requirements under given channel conditions by conserving the computational complexity and power consumption.

Finally, we compare the block error rate (BLER) of the proposed algorithm with the link adaption scheme presented in [29]. For the same simulation parameters, desired BLER of $10^{-2}$, and desired throughput of 5 Mbps, figure 12 presents BLER plotted versus SNR for both the algorithms. It can be seen that the proposed

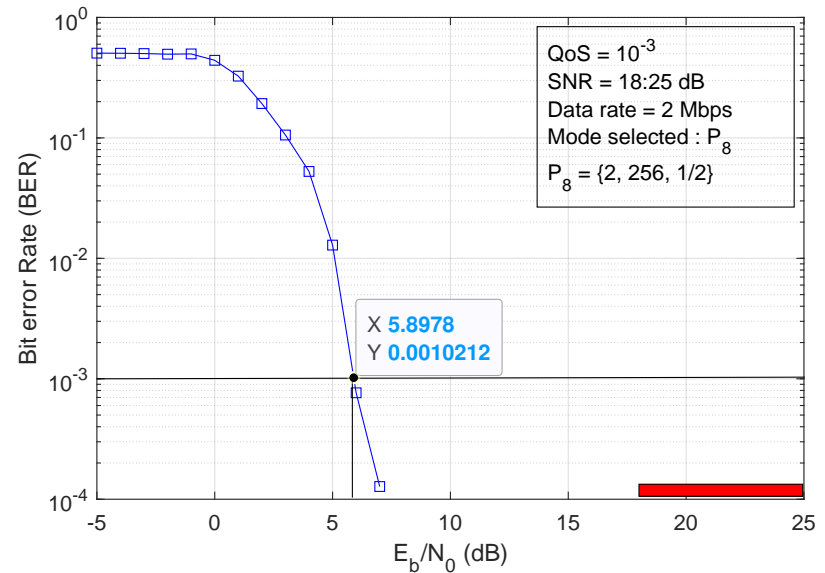

Fig. 11: Mode selection for $E_{3}, S_{4}, R_{1}$

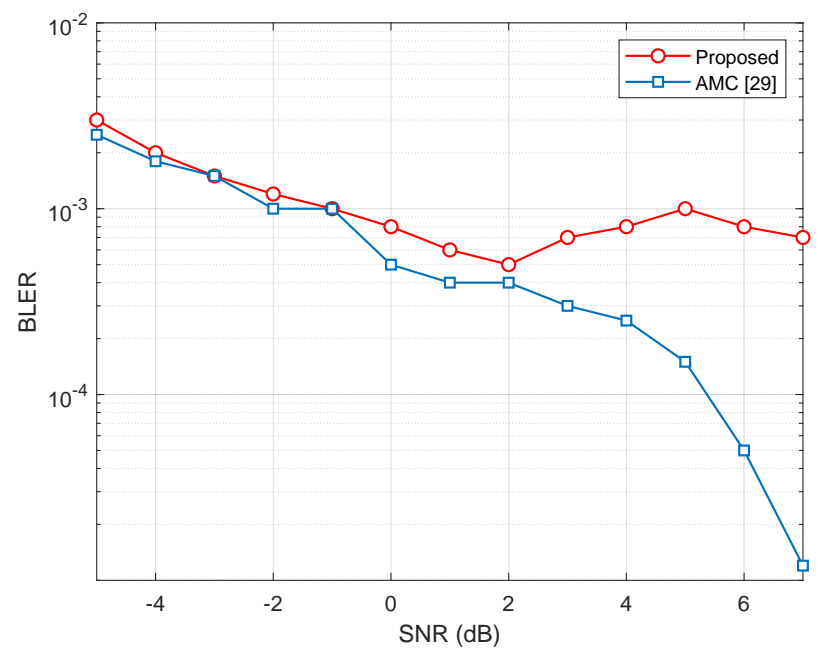

Fig. 12: BLER vs SNR for the proposed link adaptation and ACM algorithm of [29]

algorithm is superior to the other scheme because of intelligent selection of appropriate mode of operation.

\section{Conclusion}

In this paper, an efficient link adaptation algorithm for FBMC/OQAM is proposed. A throughput optimization problem with constraint on desired quality of service under a given channel condition is formulated. The proposed algorithm selects the most feasible mode of operation consisting of appropriate modulation order, number of subcarriers and code rate. The inclusion of throughput as input to the adaptive algorithm is a significant contribution. The aim is to reduce the computational complexity and power consumption by simultaneously achieving all the service requirements under 
given channel conditions. Simulation results prove the validity of the proposed scheme and affirms its superiority over existing scheme.

\section{Conflict of interest}

The authors declare that they have no conflict of interest.

\section{References}

1. S. Kundrapu, S. I. Dutt, N. K. Koilada, and A. C. Raavi, "Characteristic analysis of OFDM, FBMC and UFMC modulation schemes for next generation wireless communication network systems," in 2019 3rd International conference on Electronics, Communication and Aerospace Technology (ICECA). IEEE, 2019, pp. 715721.

2. B. Farhang-Boroujeny and R. Kempter, "Multicarrier communication techniques for spectrum sensing and communication in cognitive radios," IEEE Communications Magazine, vol. 46, no. 4, pp. 80-85, 2008.

3. M. S. John and J. Soumya, "Achieving cognitive radio for improved spectrum utilization: An implementation," 2019.

4. F. K. Jondral, "Software-defined radio-basics and evolution to cognitive radio," EURASIP journal on wireless communications and networking, vol. 2005, no. 3, p. 652784, 2005

5. R. Krishnan, R. G. Babu, S. Kaviya, N. P. Kumar, C. Rahul, and S. S. Raman, "Software defined radio (SDR) foundations, technology tradeoffs: A survey," in 2017 IEEE International Conference on Power, Control, Signals and Instrumentation Engineering (ICPCSI). IEEE, 2017, pp. 2677-2682.

6. D. Bepari and D. Mitra, "Improved power loading scheme for orthogonal frequency division multiplexing based cognitive radio," IET Communications, vol. 9, no. 16, pp. 2033-2040, 2015

7. J. Du and S. Signell, "Comparison of CP-OFDM and OFDM/OQAM in doubly dispersive channels," in Future Generation Communication and Networking (FGCN 2007), vol. 2. IEEE, 2007, pp. 207-211.

8. S. Kaur, L. Kansal, G. S. Gaba, and N. Safarov, "Survey of filter bank multicarrier (FBMC) as an efficient waveform for 5G," International Journal of Pure and Applied Mathematics, vol. 118, no. 7, pp. 45-49, 2018.

9. A. Zafar, "Filter bank based multicarrier systems for future wireless networks." Ph.D. dissertation, University of Surrey, 2018.

10. S. Patchala and M. Sailaja, "Analysis of filter bank multicarrier system for 5G communications," Int. J. Wirel. Microw. Technol.(IJWMT), vol. 9, no. 4, pp. 39-50, 2019.

11. G. S. Kishore and H. Rallapalli, "Towards 5G: A Survey on Waveform Contenders," in Advances in Decision Sciences, Image Processing, Security and Computer Vision. Springer, 2020, pp. 243-250.

12. S. H. Alrubaee, M. Ismail, M. A. Altahrawi, and B. B. Burhan, "Filter bank multi-carrier modulation technique for vehicle-to-vehicle communication [j]," Journal of Communications, vol. 15, no. 7, pp. 566-571, 2020.
13. G. Miao, N. Himayat, and G. Y. Li, "Energy-efficient link adaptation in frequency-selective channels," IEEE Transactions on communications, vol. 58 , no. 2, pp. 545$554,2010$.

14. M. Riback, S. Grant, G. Jongren, T. Tynderfeldt, D. Cairns, and T. Fulghum, "MIMO-HSPA testbed performance measurements," in 2007 IEEE 18th International Symposium on Personal, Indoor and Mobile Radio Communications. IEEE, 2007, pp. 1-5.

15. B. S. K. Reddy and B. Lakshmi, "Adaptive modulation and coding for mobile-WiMAX using SDR in GNU radio," in 2014 International Conference on Circuits, Systems, Communication and Information Technology Applications (CSCITA). IEEE, 2014, pp. 173-178.

16. W. N. W. Muhamad, S. S. Sarnin, A. Idris, and A. Syahira, "Link adaptation algorithm for ieee 802.11 wireless local area netwoks in fading channel," Indonesian Journal of Electrical Engineering and Computer Science, vol. 12 , no. 2, pp. 677-684, 2018.

17. J. Saha, S. S. Das, and S. Mukherjee, "Link adaptation using dynamically allocated thresholds and power control," Wireless Personal Communications, vol. 103, no. 3, pp. 2259-2283, 2018.

18. M. A. Alavi, W. Fernando, P. Permuna, K. Jayathilake, S. Mathurusha, N. Vithanage, and D. Dhammearatchi, "Enhanced QoS support in OFDMA-based WiMAX systems," Compusoft, vol. 5, no. 4, p. 2090, 2016.

19. B. S. K. Reddy and B. Lakshmi, "Adaptive modulation and coding with channel state information in OFDM for WiMAX," IJ Image, Graphics and Signal Processing, vol. 1, pp. 61-69, 2015.

20. L. Wan, H. Zhou, X. Xu, Y. Huang, S. Zhou, Z. Shi, and J.-H. Cui, "Adaptive modulation and coding for underwater acoustic OFDM," IEEE Journal of Oceanic Engineering, vol. 40, no. 2, pp. 327-336, 2014.

21. M. Zeeshan and S. A. Khan, "A novel fuzzy inferencebased technique for dynamic link adaptation in SDR wideband waveform," IEEE Transactions on Communications, vol. 64, no. 6, pp. 2602-2609, 2016.

22. R. S. Janaki and V. Nagarajan, "Fuzzy logic based adaptive modulation system for energy efficient wireless sensor network," 2018.

23. Z. Yang, L. Cai, A. Gulliver, L. He, and J. Pan, "Beyond powers of two: Hexagonal modulation and nonbinary coding for wireless communication systems," arXiv preprint arXiv:1712.07630, 2017.

24. H. C. Mora, N. O. Garzón, and C. de Almeida, "On the cellular spectral efficiency of MC-CDMA systems with MMSE multiuser detector employing fractional and soft frequency reuse," AEU-International Journal of Electronics and Communications, vol. 84, pp. 34-45, 2018

25. K. Shahzad, S. Gulzar, M. Zeeshan, and S. A. Khan, "A novel hybrid narrowband/wideband networking waveform physical layer for multiuser multiband transmission and reception in software defined radio," Physical Communication, vol. 36, p. 100790, 2019.

26. M. Caus, M. Navarro, X. Mestre, and A. Pérez-Neira, "Link adaptation in FBMC/OQAM systems using NBLDPC codes," in 2016 European Conference on Networks and Communications (EuCNC). IEEE, 2016, pp. 11-15.

27. M. S. Khairy, A. Khajeh, A. M. Eltawil, and F. J. Kurdahi, "Joint power management and adaptive modulation and coding for wireless communications systems with unreliable buffering memories," IEEE Transactions on Circuits and Systems I: Regular Papers, vol. 61, no. 8, pp. 2456-2465, 2014. 
28. K. Singh, "Fuzzy logic based modified adaptive modulation implementation for performance enhancement in ofdm systems," International Journal of Intelligent Systems and Applications, vol. 8, no. 5, p. 49, 2016.

29. M. Caus, M. Navarro, X. Mestre, and A. Pérez-Neira, "Link adaptation in fbmc/oqam systems using nb-ldpc codes," in 2016 European Conference on Networks and Communications (EuCNC). IEEE, 2016, pp. 11-15.

30. M. Bellanger, D. Le Ruyet, D. Roviras, M. Terré, J. Nossek, L. Baltar, Q. Bai, D. Waldhauser, M. Renfors, T. Ihalainen et al., "FBMC physical layer: a primer," Phydyas, vol. 25 , no. 4, pp. 7-10, 2010.

31. I. B. F. de Almeida, L. L. Mendes, J. J. Rodrigues, and M. A. da Cruz, "5G waveforms for IoT applications," IEEE Communications Surveys \& Tutorials, vol. 21, no. 3, pp. 2554-2567, 2019.

32. R. N. Mitra and D. P. Agrawal, " $5 \mathrm{G}$ mobile technology: A survey," ICT Express, vol. 1, no. 3, pp. 132-137, 2015.

33. S. Kundrapu, S. I. Dutt, N. K. Koilada, and A. C. Raavi, "Characteristic analysis of OFDM, FBMC and UFMC modulation schemes for next generation wireless communication network systems," in 2019 3rd International conference on Electronics, Communication and Aerospace Technology (ICECA). IEEE, 2019, pp. 715721.

34. A. F. Isnawati, V. O. Citra, and J. Hendry, "Performance analysis of audio data transmission on FBMCOffset QAM system," in 2019 IEEE International Conference on Industry 4.0, Artificial Intelligence, and Communications Technology (IAICT). IEEE, 2019, pp. 8186.

35. A. I. Pérez-Neira, M. Caus, R. Zakaria, D. Le Ruyet, E. Kofidis, M. Haardt, X. Mestre, and Y. Cheng, "MIMO signal processing in offset-QAM based filter bank multicarrier systems," IEEE Transactions on Signal Processing, vol. 64, no. 21, pp. 5733-5762, 2016.

36. R. Jain, "Channel models: A tutorial," in WiMAX forum $A A T G$, vol. 10, 2007.

37. R. Kadel, N. Islam, K. Ahmed, and S. J. Halder, "Opportunities and challenges for error correction scheme for wireless body area network-a survey," Journal of Sensor and Actuator Networks, vol. 8, no. 1, p. 1, 2019.

38. F. Sandoval, G. Poitau, and F. Gagnon, "Optimizing forward error correction codes for COFDM with reduced PAPR," IEEE Transactions on Communications, vol. 67 , no. 7, pp. 4605-4619, 2019.

39. S. Garg, A. K. Sharma, and A. Tyagi, "Performance analysis of $1 / 2$ code rate convolution encoded dwt-ofdm system with different modulation schemes," Intl. J. Innovative Res. Sci. Eng, vol. 3, pp. 109-119, 2017.

40. S. Dhaliwal, N. Singh, and G. Kaur, "Performance analysis of convolutional code over different code rates and constraint length in wireless communication," in 2017 International Conference on I-SMAC (IoT in Social, Mobile, Analytics and Cloud)(I-SMAC). IEEE, 2017, pp. $464-468$

41. V. H. S. Le, C. A. Nour, E. Boutillon, and C. Douillard, "Dual trellis construction for high-rate punctured convolutional codes," in 2019 IEEE 30th International Symposium on Personal, Indoor and Mobile Radio Communications (PIMRC Workshops). IEEE, 2019, pp. 1-7.

42. C. L. Taylor, Punctured convolutional coding scheme for multi-carrier multi-antenna wireless systems. Electronics Research Laboratory, College of Engineering, University of ..., 2001.

43. J. Du and S. Signell, "Comparison of CP-OFDM and OFDM/OQAM in doubly dispersive channels," in Future
Generation Communication and Networking (FGCN 2007), vol. 2. IEEE, 2007, pp. 207-211.

44. B. Ahmed, M. Zeeshan, and S. Gulzar, "An efficient algorithm for cfo estimation in fbmc and gfdm under sui channel models," in 2019 IEEE Latin-American Conference on Communications (LATINCOM). IEEE, 2019, pp. 1-6.

45. F. Sandoval, G. Poitau, and F. Gagnon, "Optimizing forward error correction codes for cofdm with reduced papr," IEEE Transactions on Communications, vol. 67, no. 7, pp. 4605-4619, 2019. 
Figures

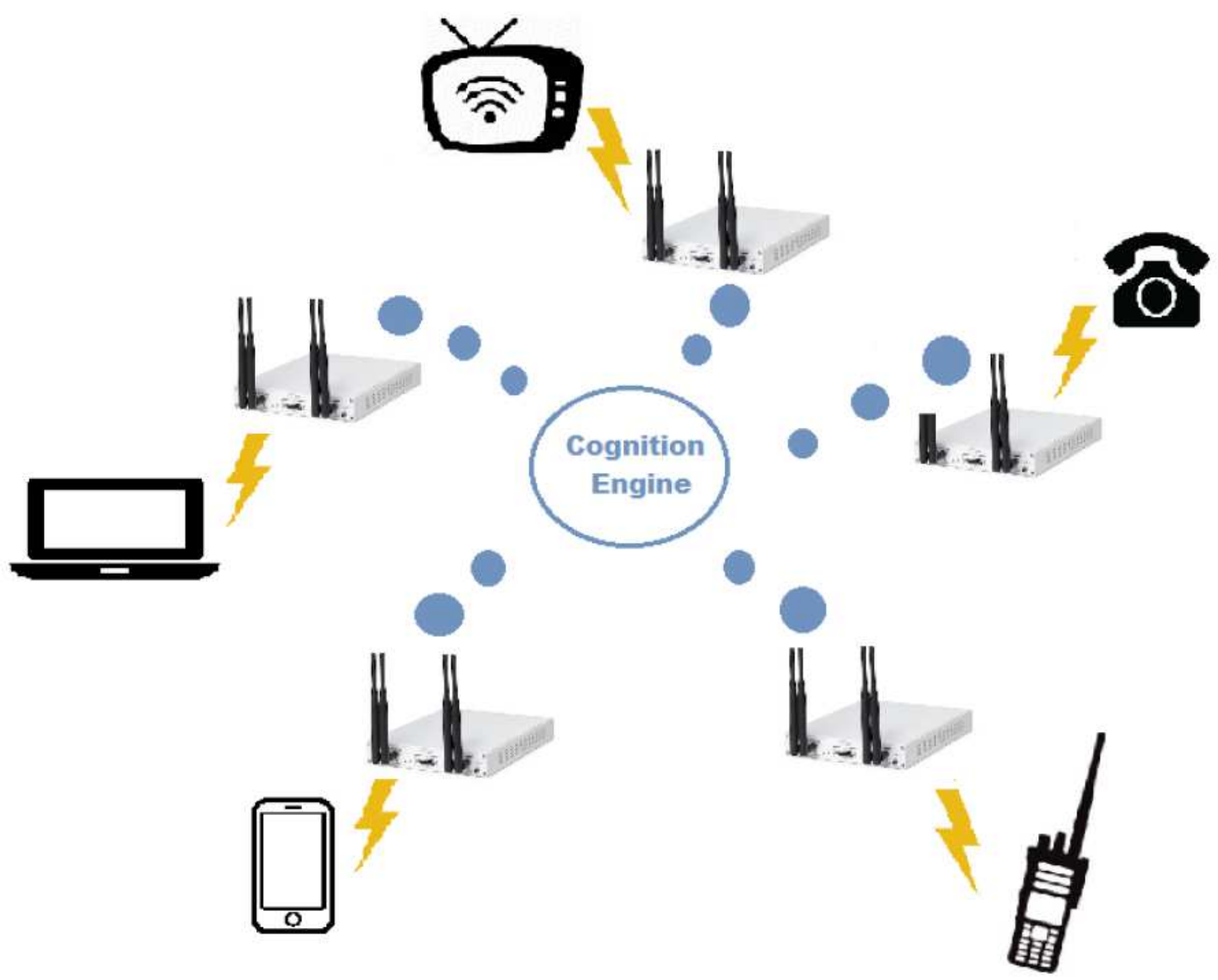

Figure 1

Networked demonstration for various QoS applications 


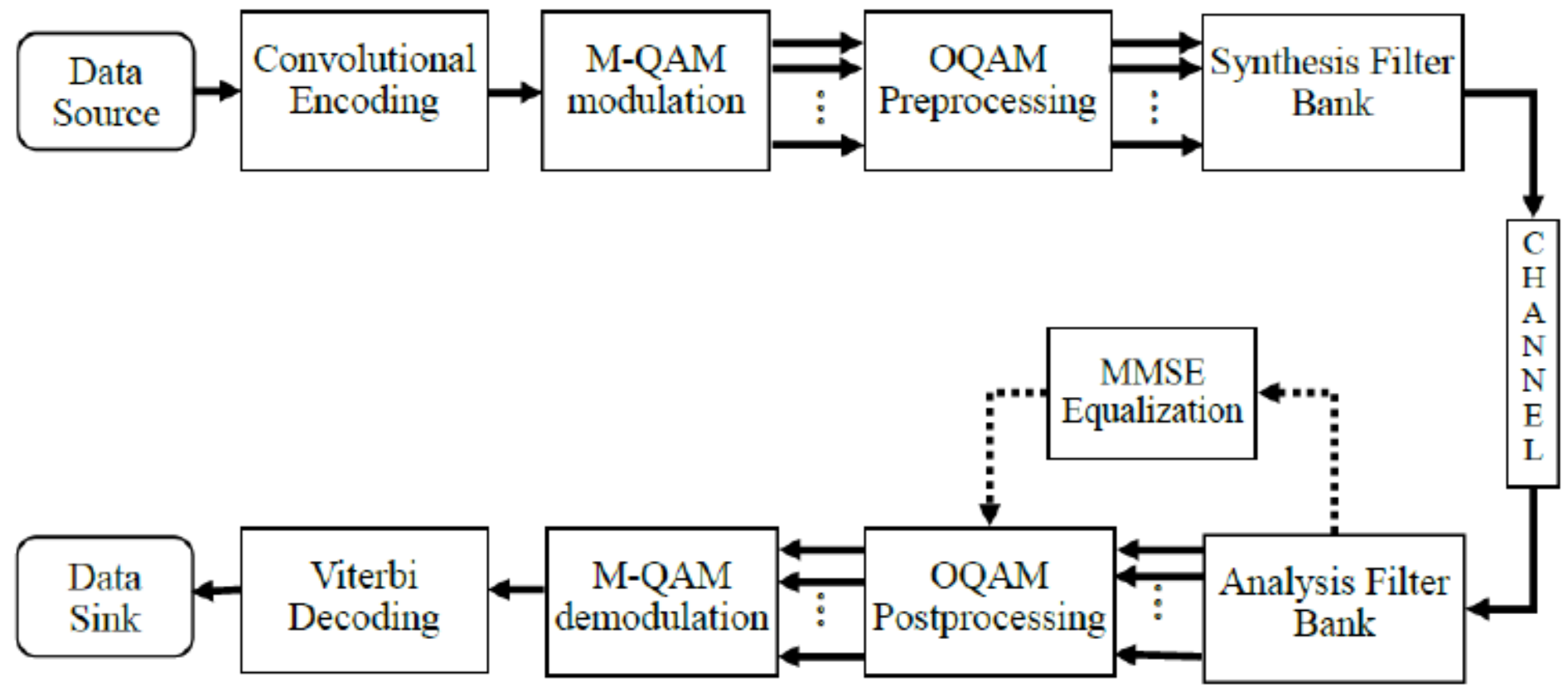

Figure 2

FBMC/OQAM - System Model 


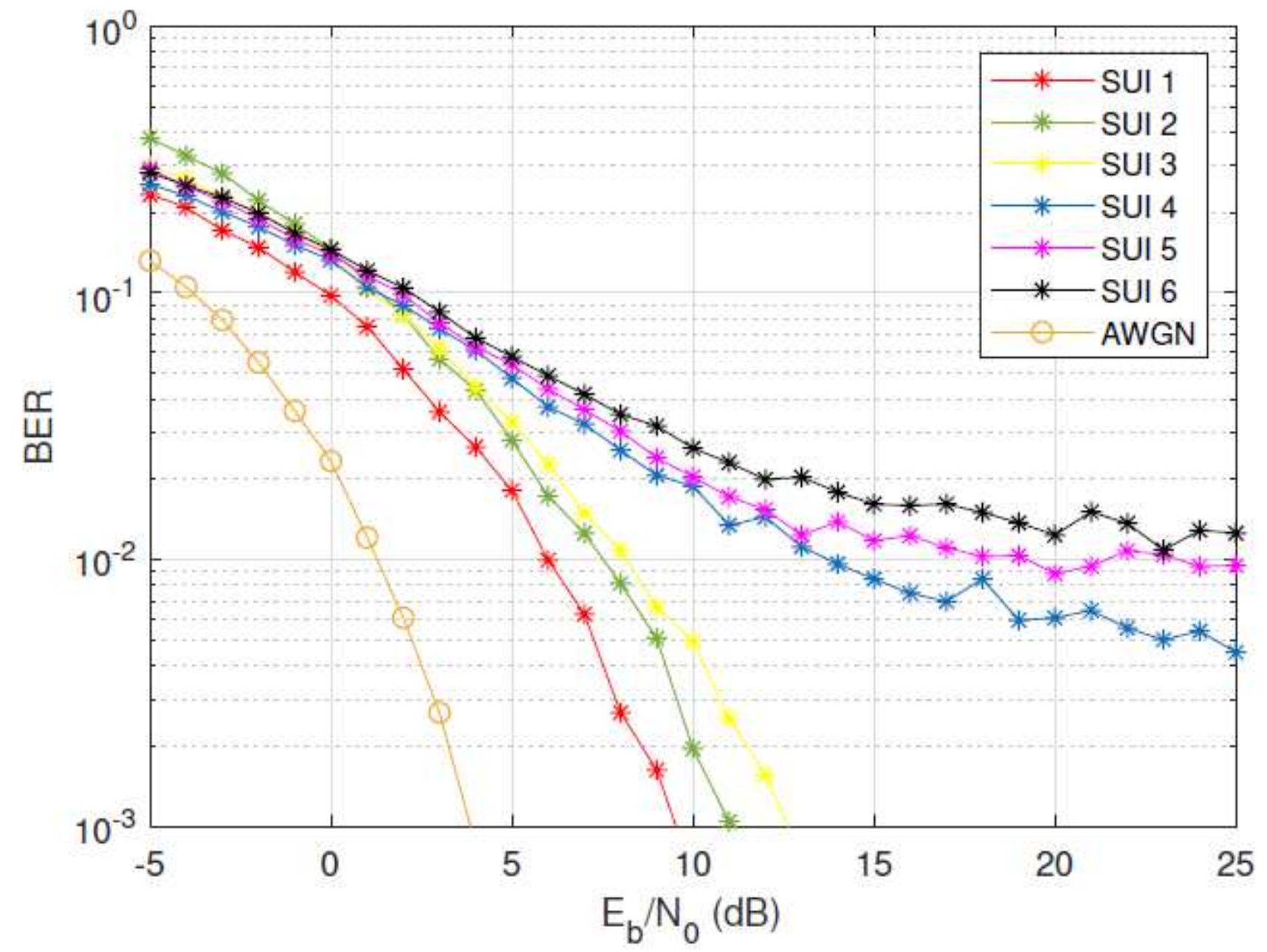

Figure 3

FBMC/QAM without convolutional encoding 


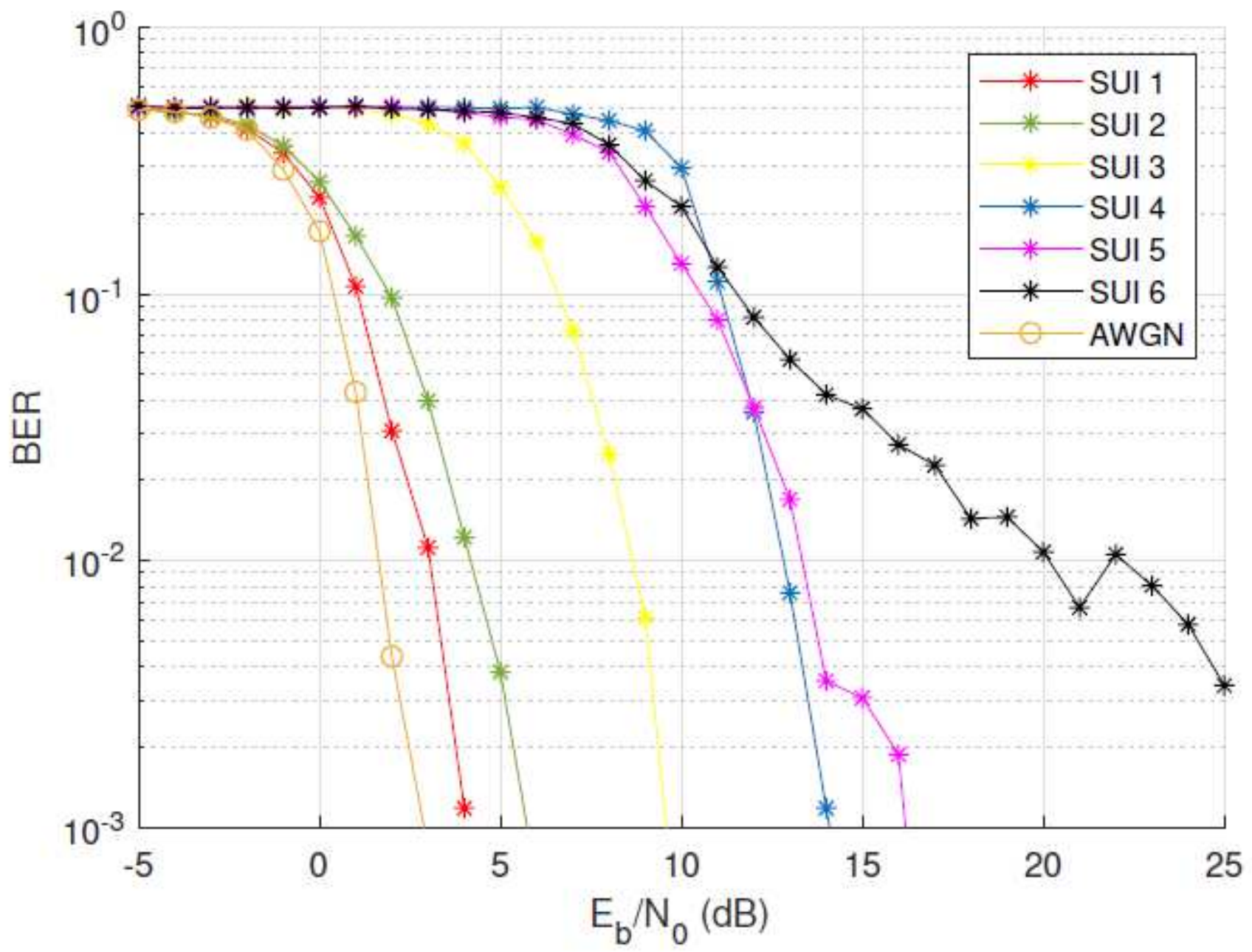

Figure 4

FBMC/QAM with convolutional encoding 


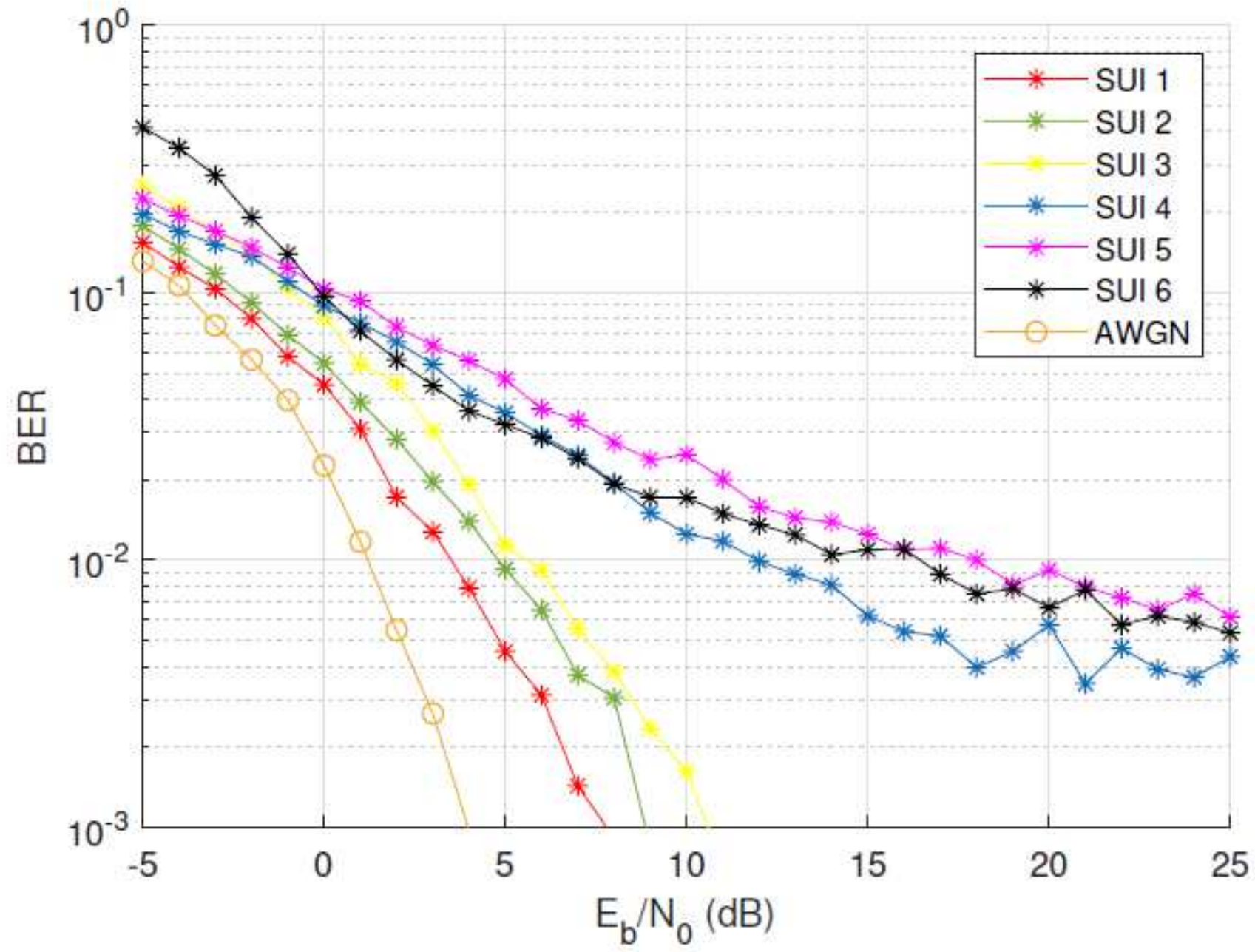

Figure 5

FBMC/OQAM without convolutional encoding 


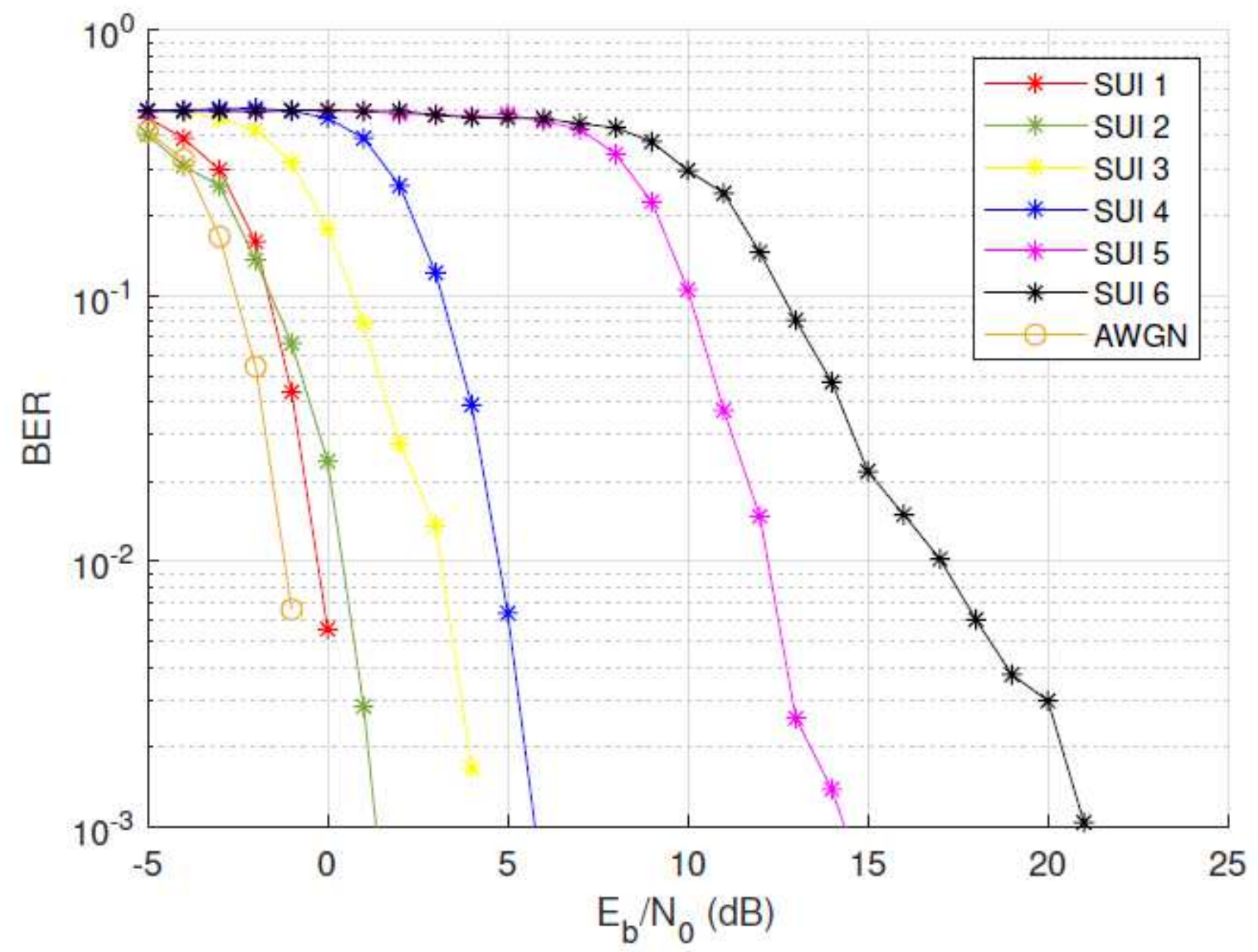

Figure 6

FBMC/OQAM with convolutional encoding

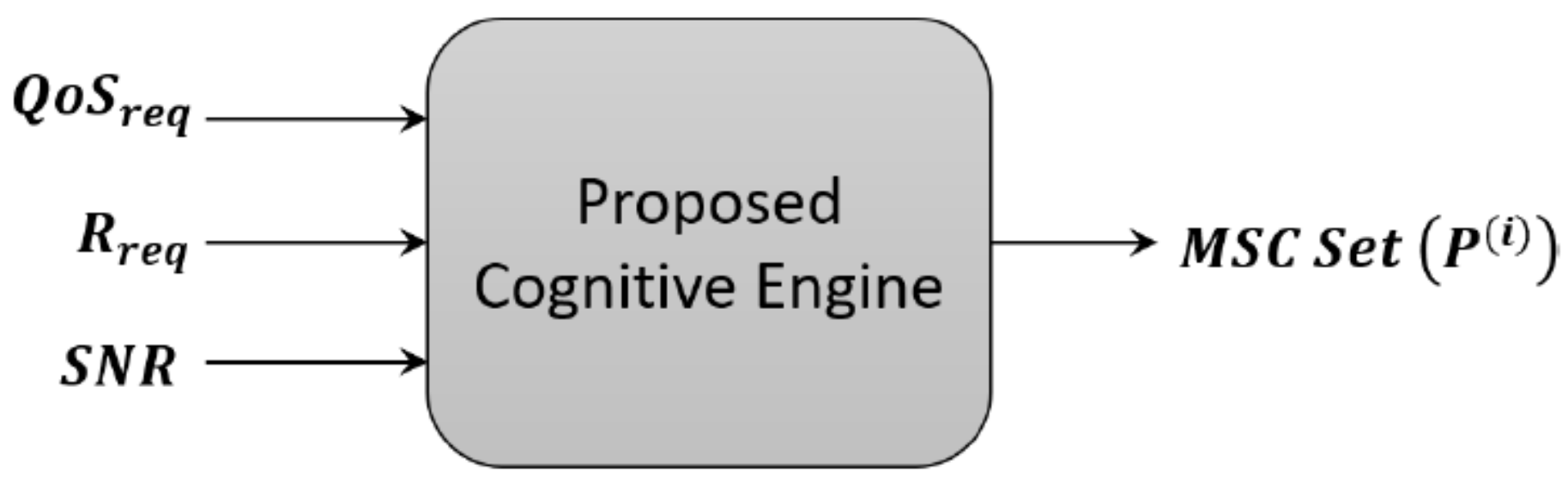

Figure 7

Concept of cognitive engine 


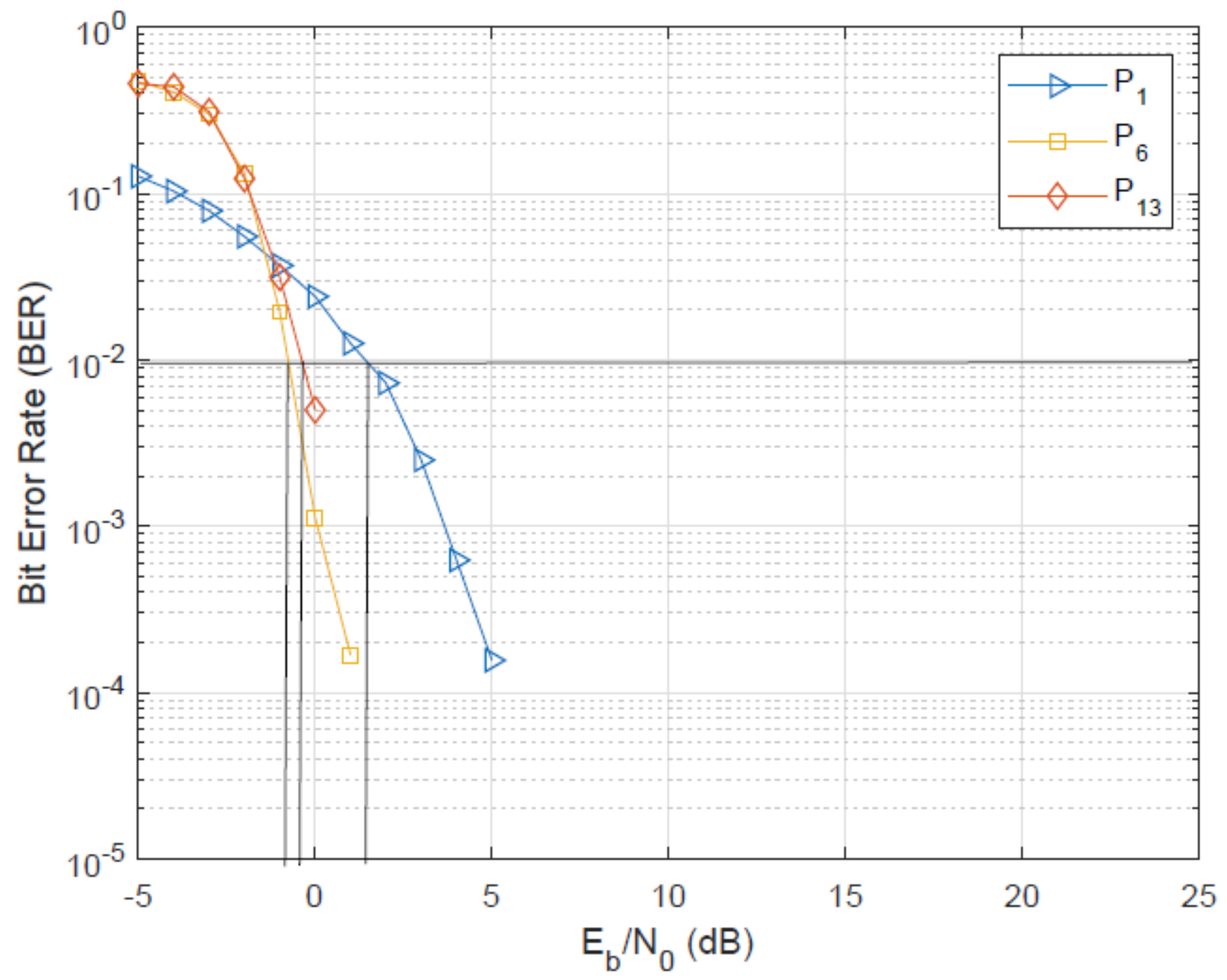

Figure 8

Data Acquisition for various modes of operation in AWGN 


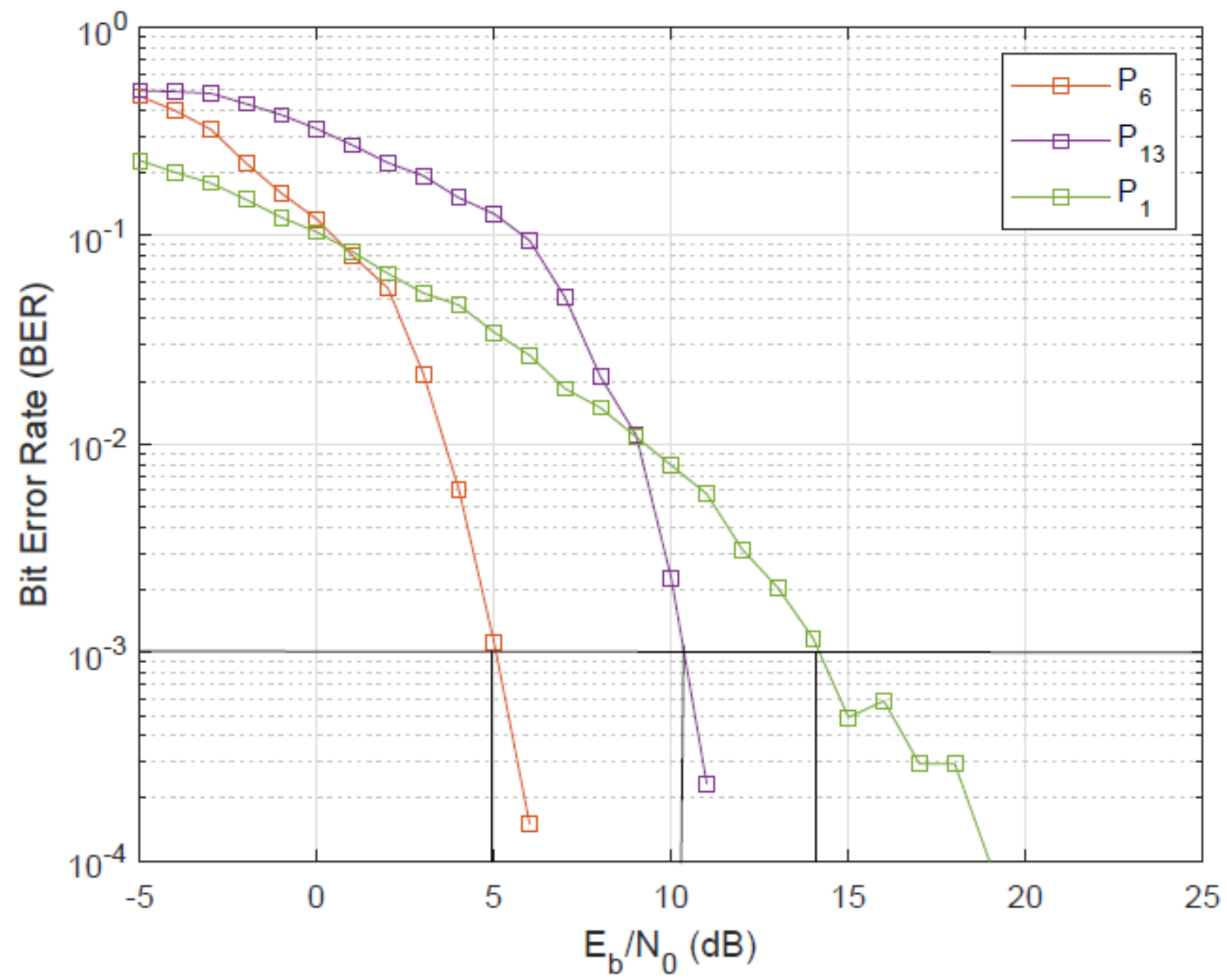

Figure 9

Data Acquisition for various modes of operation in SUI-4 


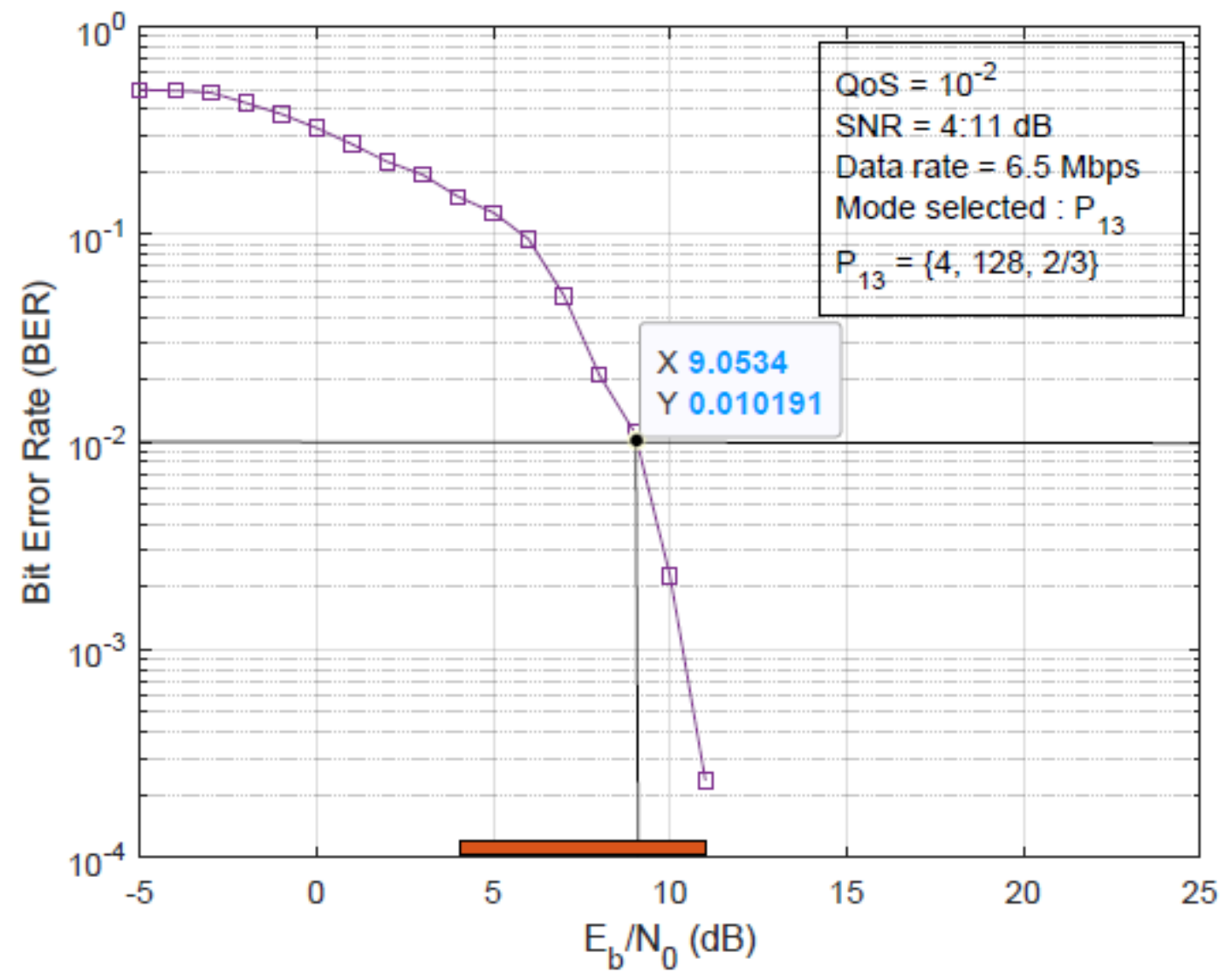

Figure 10

Mode selection for E2, S2, R3 


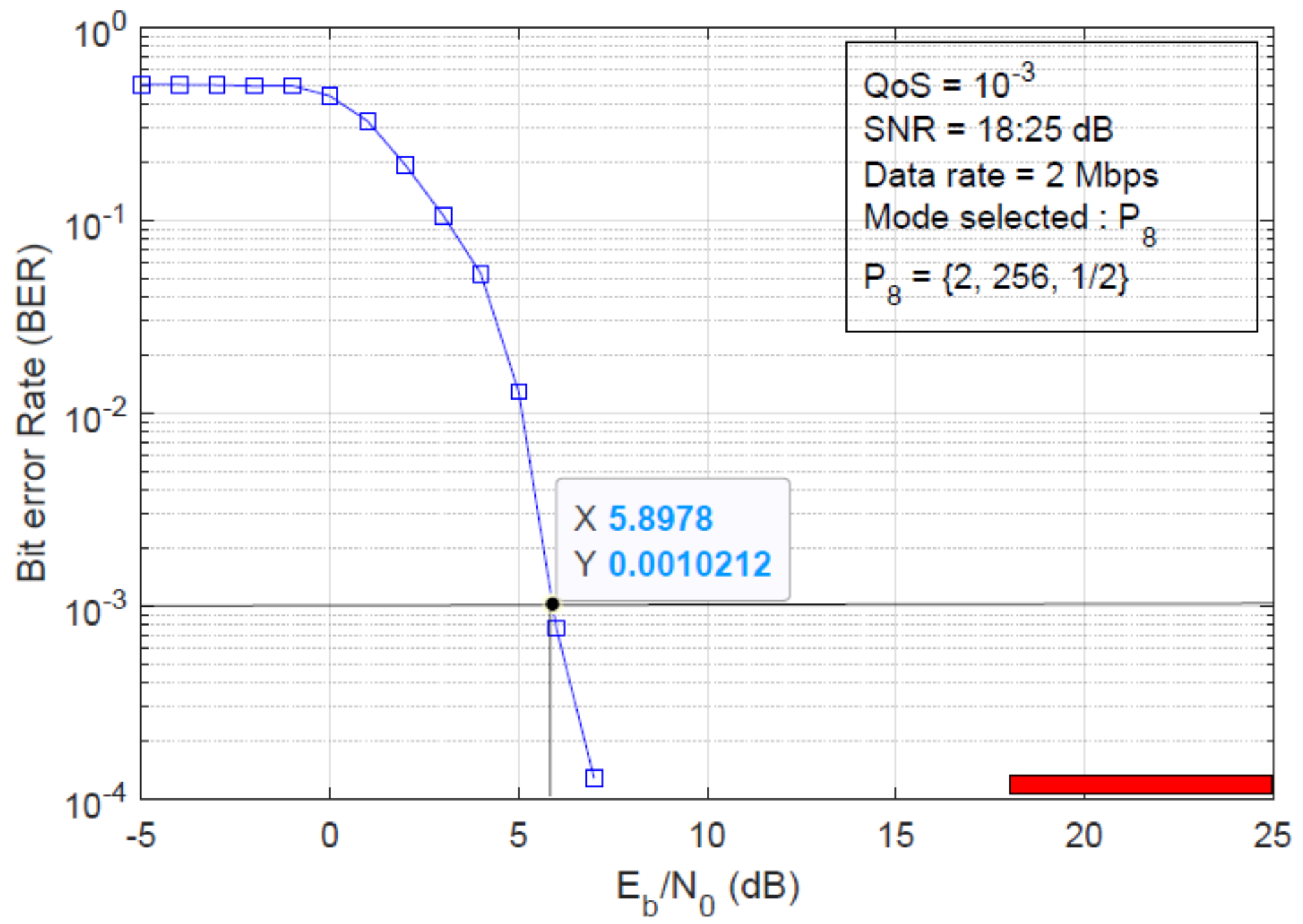

Figure 11

Mode selection for E3, S4, R1 


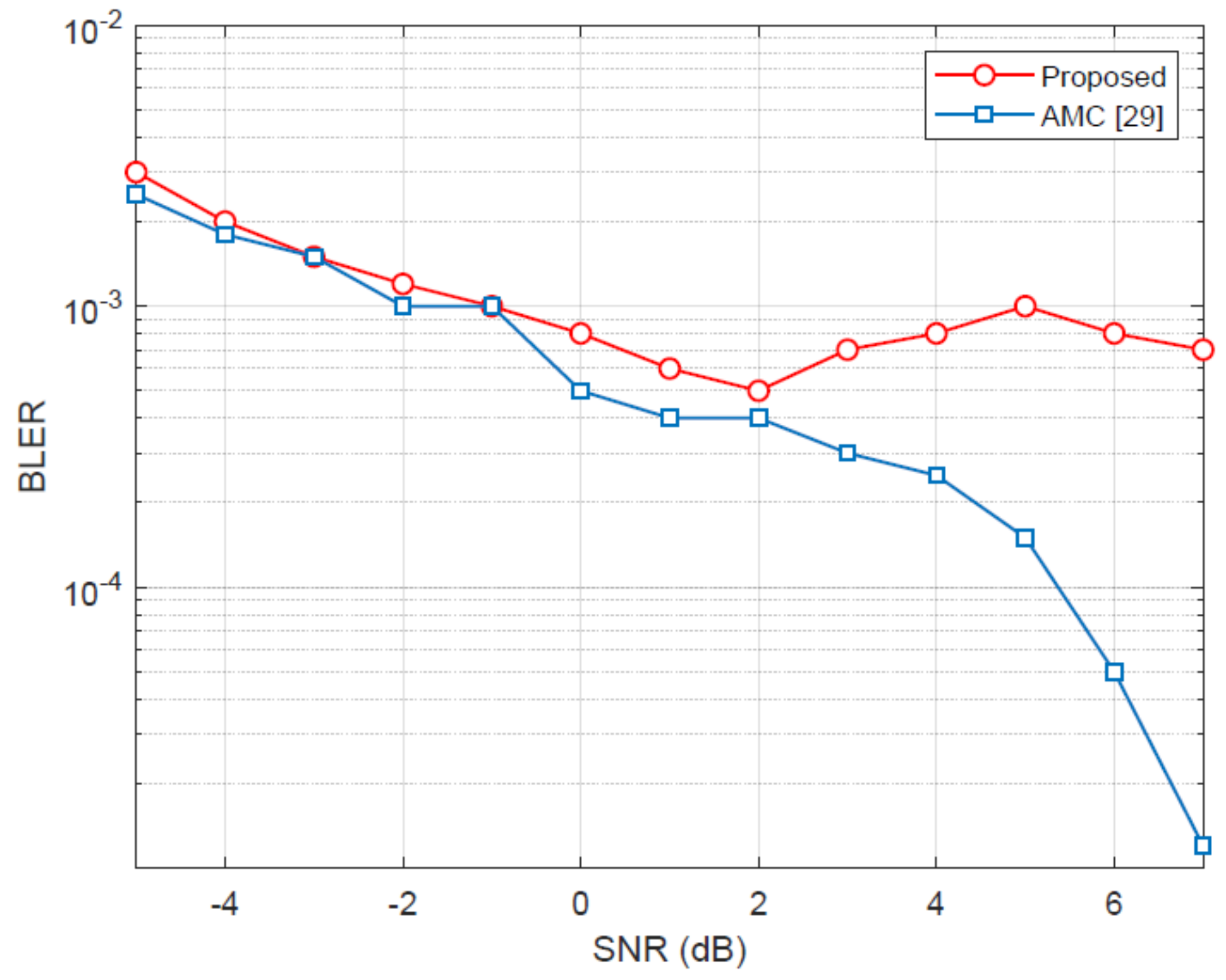

Figure 12

BLER vs SNR for the proposed link adaptation and ACM algorithm of [29] 